* * Published in Journal of Mathematical Physics, vol. 42, no. 4, pp. 1667-1688, 2001 


\title{
Systems of Reaction Diffusion Equations and their Symmetry Properties
}

\author{
A.G. Nikitin ${ }^{\dagger}$, R.J.Wiltshire ${ }^{\ddagger} *$ \\ ${ }^{\dagger}$ Institute of Mathematics, National Academy of Sciences \\ of the Ukraine, 3 Tereshchenkivska Street, Kiev 4, \\ Ukraine \\ $\ddagger$ Division of Mathematics, School of Technology, \\ University of Glamorgan, Pontypridd, CF37 1DL, UK
}

\begin{abstract}
A constructive algorithm is proposed for the investigation of symmetries of partial differential equations. The algorithm is used to present classical Lie symmetries of systems of two non-linear reaction diffusion equations.
\end{abstract}




\section{Introduction}

Mathematical models which ultimately involve the analysis of coupled systems of nonlinear diffusion equations are often discussed in the literature. The Schrödinger equation in $m$-dimensional space is an obvious example from mathematical physics whilst activatorinhibitor reactions [1], predator-prey systems, $\lambda-\omega$ reaction systems [2] are common examples from mathematical biology. These systems are very complex in nature and admit fundamental particular solutions (for example, travelling waves and, spiral waves) which have a clear group-theoretical interpretation and which can be obtained using the classical Lie approach. The existence of such solutions suggests an important role for both the classical and non-classical symmetry analysis of systems of reaction diffusion equations. However, to the best of our knowledge, a comprehensive group analysis has not been undertaken previously although analyses of some special cases do exist.

In this paper we discuss symmetries of equations in the general form

$$
\frac{\partial u}{\partial t}-A \sum_{i} \frac{\partial^{2} u}{\partial x_{i}^{2}}-f(u)=0
$$

where $u \in \mathbb{R}^{n}, f \in \mathbb{R}^{n},(x, t) \in \mathbb{R}^{m} \times \mathbb{R}$ and $A$ is a $n \times n$ constant non-singular matrix. Actually we restrict ourselves to the case $n=2$ but all results present in Section 2 and the main part of results of Section 3 are valid for arbitrary $n$.

We note that equation (1) with $m=n=1, f \equiv 0$ was the subject of a group analysis by Sophus Lie [3]. In addition, classical Lie symmetries of equation (1) with, $n=m=1$, were investigated by Ovsiannikov [4] whose results were completed by Dorodnitsyn [5] and then generalized to the case $m=2,3$ by Dorodnitsyn, Kniazeva and Svirishchevski [6]. The related conditional (nonclassical) symmetries were described by Fushchych and Serov [7] and Clarkson and Mansfield [8]. The classical symmetries are summarized in Table 1 .

Table 1: Symmetries of the scalar diffusion equation with source

\begin{tabular}{|c|c|}
\hline$f(u)$ & Infinitesimals Generator, $X$ \\
\hline arbitrary & $X_{0}$ \\
\hline 0 & $X_{0}+\nu G_{0}+\mu \widehat{D}_{0}+\lambda \hat{A}_{0}+\sigma u \frac{\partial}{\partial u}+\psi_{0} \frac{\partial}{\partial u}$ \\
\hline 1 & $X_{0}+\nu G_{1}+\mu \widehat{D}_{1}+\lambda \widehat{A}_{1}+\sigma(u-t) \frac{\partial}{\partial u}+\psi_{0} \frac{\partial}{\partial u}$ \\
\hline$u$ & $X_{0}+\nu G_{0}+\mu \widehat{D}_{2}+\alpha \hat{A}_{2}+\sigma u \frac{\partial}{\partial u}+\psi_{1} \frac{\partial}{\partial u}$ \\
\hline$u^{n}, n \neq 1$ & $X_{0}+\mu \widehat{D}_{3}$ \\
\hline$e^{u}$ & $X_{0}+\mu \widehat{D}_{4}$ \\
\hline$f=u \ln u$ & $X_{0}+\nu e^{t}\left(\frac{\partial}{\partial x}-\frac{1}{2} x u \frac{\partial}{\partial u}\right)+\mu e^{t} u \frac{\partial}{\partial u}$ \\
\hline
\end{tabular}

Here Greek letters denote arbitrary parameters and are the translation, $X_{0}$; Galilei, $G_{\alpha}(\alpha=0,1)$; scale, $\widehat{D}_{\mu}(\mu=0,1, \ldots, 4)$ and conformal, $\hat{A}_{s}(\sigma=0,1,2)$ generators respectively, where :

$$
\begin{gathered}
X_{0}=\alpha \frac{\partial}{\partial t}+\beta \frac{\partial}{\partial x}, \quad G_{\alpha}=t \frac{\partial}{\partial x}-\frac{1}{2} x u \frac{\partial}{\partial u}+\frac{1}{2} \delta_{\alpha 1} t x \frac{\partial}{\partial u}, \quad \widehat{D}_{0}=2 t \frac{\partial}{\partial t}+x \frac{\partial}{\partial x}, \\
\widehat{D}_{1}=\widehat{D}_{0}+2 t \frac{\partial}{\partial u}, \quad \widehat{D}_{2}=\widehat{D}_{0}+2 t u \frac{\partial}{\partial u}, \quad \widehat{D}_{3}=\widehat{D}_{0}-\frac{2}{n-1} u \frac{\partial}{\partial u}, \\
\widehat{D}_{4}=D_{0}-2 \frac{\partial}{\partial u}, \quad \hat{A}_{\mu}=\frac{1}{2} t \widehat{D}_{\mu}-\frac{1}{4}\left(x^{2}+2 t\right)\left(1-\delta_{\mu 1} t\right) u \frac{\partial}{\partial u} .
\end{gathered}
$$


In addition, $\delta_{\mu \nu}$ is the Kronecker symbol and $\psi_{n}(n=0,1)$ is an arbitrary solution of the linear diffusion equation $\left(\frac{\partial}{\partial t}-\frac{\partial^{2}}{\partial x^{2}}\right) \psi_{n}=n \psi_{n}$.

A systematic investigation of the symmetries of the general equation (1) with $m>1$ and $n>1$ has so far not been considered in detail although partial results are available in [10], [11], [9], [2]. In recent paper [12] Lie symmetries of a subclass of systems (1) for $n=2$ and arbitrary $m$ were investigated. In this subclass the matrix $A$ was diagonal but not a multiple of the unit matrix. We shall demonstrate that the results [12] are incomplete.

It is the aim here to undertake such an investigation for the most general case of an arbitrary $2 \times 2$ matrix $A$. In this way we present a complete description of Lie symmetries of all possible systems (1) including the cases of the unit matrix $A$. This latter case corresponds to the most rich of symmetries with many interesting applications. In particular, we find all nonlinear Schrödinger equations admitting nontrivial Lie symmetries since these equations can also be represented in the form (1) with an antidiagonal matrix $A$, as is demonstrated in Section 7. Moreover, we find new symmetries of (1) also in the subclass studied in [12].

The additional main aim of this paper is to present the novel use of a rather conventional algorithm for investigation of symmetries of a special class of partial differential equations which includes (1) as a particular case. More precisely we will show that the classical Lie approach (refer, for example, [13],[11]) when applied to systems (1) admits a rather simple formulation in terms of commutator algebra which may also be applied to extended classes of partial differential equations. Furthermore the algorithm may be used to determine non-classical (or, conditional) symmetries of (1). It will be shown that for $n>1$ there is a proliferation of symmetries, including for the case when $f(u)$ is linear which do not have origins in symmetries of Table 1 for the one dimensional scalar diffusion equation with source.

\section{An Algorithm for the Determining equations of symmetries for the system (1)}

We require form-invariance of the system of reaction diffusion equations (1) with respect to the one-parameter group of transformations:

$$
t \rightarrow t^{\prime}(t, x, \varepsilon), \quad x \rightarrow x^{\prime}(t, x, \varepsilon), \quad u \rightarrow u^{\prime}\left(t^{\prime}, x^{\prime}, \varepsilon\right),
$$

where $\varepsilon$ is a group parameter. In other words, we require that $u^{\prime}\left(t^{\prime}, x^{\prime}, \varepsilon\right)$ satisfies the same equation as $u(t, x)$ :

$$
L^{\prime} u^{\prime}=f\left(u^{\prime}\right), \quad L^{\prime}=\frac{\partial}{\partial t^{\prime}}-A \sum_{i} \frac{\partial_{i}^{2}}{\partial x_{i}^{\prime 2}} .
$$

From the infinitesimal transformations:

$$
\begin{aligned}
& t \rightarrow t^{\prime}=t+\Delta t=t+\varepsilon \eta, \quad x_{a} \rightarrow x_{a}^{\prime}=x_{a}+\Delta x_{a}=x_{a}+\varepsilon \xi^{a}, \\
& u_{a} \rightarrow u_{a}^{\prime}=u_{a}+\Delta u_{a}=u_{a}+\varepsilon \pi_{a}
\end{aligned}
$$


we obtain the following representation for the operator $L^{\prime}$ :

$$
L^{\prime}=\left[1+\varepsilon\left(\eta \frac{\partial}{\partial t}+\xi^{a} \frac{\partial}{\partial x_{a}}\right)\right] L\left[1-\varepsilon\left(\eta \frac{\partial}{\partial t}+\xi^{a} \frac{\partial}{\partial x_{a}}\right)\right]+O\left(\varepsilon^{2}\right) .
$$

Using the classical Lie algorithm it is possible to find the determining equations for the functions $\eta, \xi_{a}$ and $\pi_{a}$ which specify the generator $X$ of the symmetry group:

$$
X=\eta \frac{\partial}{\partial t}+\xi^{a} \frac{\partial}{\partial x_{a}}-\pi^{b} \frac{\partial}{\partial u_{b}}
$$

where a summation from 1 to $m$ and from 1 to $n$ is assumed over repeated indices $a$ and $b$ respectively. This system will not be reproduced here but we note that three of the equations are:

$$
\frac{\partial \eta}{\partial u_{a}}=0, \quad \frac{\partial \xi^{a}}{\partial u_{b}}=0, \quad \frac{\partial^{2} \pi^{a}}{\partial u_{c} \partial u_{b}}=0 .
$$

So from (8) $\eta$ and $\xi^{a}$ are functions of $t$ and $x_{a}$ and, $\pi^{a}$ is linear in $u_{a}$. Thus:

$$
\pi^{a}=-\pi^{a b} u_{b}-\omega^{a}
$$

where $\pi^{a b}$ and $\omega^{a}$ are functions of $t$ and $x=\left(x_{1}, x_{2}, \ldots x_{m}\right)$.

From (4) it is possible to deduce all the remaining determining equations. Indeed, substituting (5), (9) into (6), using (1) and neglecting the terms of order $\varepsilon^{2}$ we find that:

$$
[Q, L] u-L \omega=\pi f+\frac{\partial f}{\partial u_{a}}\left(-\pi^{a b} u_{b}-\omega^{a}\right), \quad Q=\eta \frac{\partial}{\partial t}+\xi^{a} \frac{\partial}{\partial x_{a}}+\pi
$$

and $\pi$ is a matrix whose elements $\pi^{a b}$ are defined by the relation (9).

To guarantee that equation (10) is compatible with (1) and does not impose new nontrivial conditions for $u$ in addition to (1) it is necessary to suppose that the commutator $[Q, L]$ admits the representation:

$$
[Q, L]=\Lambda L+\varphi(t, x)
$$

where $\Lambda$ and $\varphi$ are $n \times n$ matrices dependent on $\left(t, x_{a}\right)$.

Substituting (11) into (10) the following determining equations for $f$ are obtained:

$$
\left(\Lambda^{k b}-\pi^{k b}\right) f^{b}+\varphi^{k b} u^{b}-(L \omega)^{k}=-\left(\omega^{a}+\pi^{a b} u_{b}\right) \frac{\partial f^{k}}{\partial u^{a}}
$$

Thus, to find all non-linearities $f^{k}$ generating Lie symmetries for equation (1) it is necessary to solve the operator equation (11) for $L, Q$ given in (4), (10) and determine the corresponding matrices $\Lambda, \pi, \varphi$ and functions $\eta$ and $\xi$. In the second step the nonlinearities $f^{a}$ may be found by solving the system of first order equations (12) with their known coefficients.

Equation (11) is a straightforward generalization of the invariance condition for the linear system of diffusion equations (1) with $f(u)=0$, so that $[Q, L]=\Lambda L$, which may readily be solved. By means of this "linearization" the problem of investigating 
symmetries of systems of nonlinear diffusion equations is reduced to the rather simple application of elements of matrix calculus in order to classify non-equivalent solutions of the determining equations.

We notice that this approach is valid for the extended class of equations $L u=f(u)$ where $L$ is a linear differential operator with constant coefficients and where $u \in \mathbb{R}^{n}$. We note also that calculations of the non-classical (conditional) symmetries for the system (1) may be reduced to the solution of the determining equations (12) where now $\Lambda, \pi, \varphi$, $\eta$ and $\xi$ are defined as solutions of the following relationship:

$$
[Q, L]=\Lambda L+\varphi(t, x)+\mu(t, x) Q
$$

and where $\mu(t, x)$ is an unknown function of the independent variables.

\section{The Symmetry Operators and their Simplification}

We now determine the general solutions for matrices $\Lambda, \varphi, \pi$ and also the functions $\xi, \eta$, $\pi$ which satisfy (12), (11).

Evaluating the commutator in (11) and equating the coefficients for linearly independent differential operators we obtain the five determining equations:

$$
\begin{aligned}
& A\left(\xi_{b}^{a}+\xi_{a}^{b}\right)=-\delta_{a b}(\Lambda A+[A, \pi]), \quad \dot{\eta}_{a}=0, \quad \dot{\eta}=\Lambda, \\
& \dot{\xi}^{a}-2 A \pi_{a}-A \xi_{n n}^{a}=0, \quad \varphi=A \pi_{n n}-\dot{\pi} .
\end{aligned}
$$

Here the dots denotes derivatives with respect to $t$ and subscripts denote derivatives with respect to the spatial variables, so for example, $\eta_{a}=\frac{\partial \eta}{\partial x_{a}}$.

From (14) $\Lambda$ is proportional to the unit matrix, $\Lambda=\lambda I$ and from (14) $[A, \pi]=0$. Indeed, choosing $a=b$ we obtain

$$
\pi-A^{-1} \pi A=\left(2 \xi_{a}^{a}-\lambda\right) I .
$$

The trace of the left hand side of (16) is equal to zero, and so $2 \xi_{a}^{a}-\lambda \equiv 0$ and $A \pi-\pi A=0$.

Equations (14)-(15) contain matrices which commute, and so they may easily be integrated using, for example, the method of characteristics. The general solution of (14)-(15) is:

$$
\begin{array}{ll}
\xi^{a}=C^{[a b]} x_{b}+\dot{d} x^{a}+g^{a}, & \eta=-2 d, \\
\pi=\frac{1}{2} A^{-1}\left(\frac{\ddot{d}}{2} x^{2}+\dot{g}^{a} x^{a}\right)+C, & \Lambda=-2 \dot{d} I, \\
\varphi=\frac{m}{2} \ddot{d}-\dot{C}-\frac{1}{2} A^{-1}\left(\frac{\dddot{d}}{2} x^{2}+\ddot{g}^{a} x^{a}\right) &
\end{array}
$$

where $d, g^{a}$ are arbitrary functions of $t$ and $C$ is a $t$-dependent matrix commuting with $A$.

By considering the $x$-dependence of functions (17) it is convenient to represent still unknown functions $\omega_{a}$, occurring in (12), as:

$$
\omega_{a}=\omega_{2}^{a} x^{2}+\omega_{1}^{a b} x_{b}+\omega_{0}^{a}+\mu^{a}
$$


where $\omega_{2}^{a}, \omega_{1}^{a b}, \omega_{0}^{a}$ are functions of $t$, and $\mu^{a}$ is a function of $t$ and $x$. Without loss of generality we suppose that all terms in the right hand side of (18) are linearly independent. Then comparing with $(12),(17)$ the functions $\mu^{k}$ have to satisfy:

$$
(L \mu)^{k}=\lambda^{k b} \mu^{b}+\xi_{0}^{k}+\xi_{1}^{k b} x_{b}+\xi_{2}^{k} x^{2}
$$

where $\lambda^{k b}$ are constants and $\xi_{0}^{k}, \xi_{1}^{k b}, \xi_{2}^{k}$ are functions of $t$.

The final step is to substitute (17), (19) into (12) and equate coefficients for all different powers of $x_{a}$. As a result we obtain the system of equations:

$$
\begin{aligned}
& \ddot{d}\left(A^{-1}\right)^{k b} f^{b}+\dddot{d}\left(A^{-1}\right)^{k b} u^{b}-\ddot{d}\left(A^{-1}\right)^{a b} u^{b} \frac{\partial f^{k}}{\partial u^{a}}=4\left(\dot{\omega}_{2}^{k}+\xi_{2}^{k}-\omega_{2}^{b} \frac{\partial f^{k}}{\partial u_{b}}\right), \\
& \dot{g}^{a}\left(A^{-1}\right)^{k b} f^{b}+\ddot{g}^{a}\left(A^{-1}\right)^{k b} u^{b}-\dot{g}^{a}\left(A^{-1}\right)^{k b} u^{b} \frac{\partial f^{k}}{\partial u^{a}}=2\left(\dot{\omega}_{1}^{k a}+\xi_{1}^{k a}-\omega_{1}^{b a} \frac{\partial f^{k}}{\partial u_{a}}\right), \\
& 2 \dot{d} f^{k}-C^{k b} f^{b}-\left(\dot{C}^{k b}+\frac{m}{2} \ddot{d} \delta^{k b}\right) u^{b}+\left(\omega_{0}^{a}+C^{a b} u^{b}\right) \frac{\partial f^{k}}{\partial u_{a}}=\dot{\omega}_{0}^{k}-2 m A^{k b} \omega_{2}^{b}+\xi_{0}^{k}, \\
& \frac{\partial f^{k}}{\partial u_{b}} \mu^{b}=\lambda^{k b} \mu^{b} .
\end{aligned}
$$

Thus, the general form of symmetry group generators for equation (1) is given by relations (7), (17),(18) where $d, g^{a}, C^{a b}, \omega_{0}^{k}, \omega_{1}^{k b}, \omega_{2}^{a}, \mu^{a}$ are functions of $t$ to be specified using equations (20)-(23). These results are valid for equation (1) with arbitrary $n \times n$ constant nonsingular matrix $A$.

In the following we restrict ourselves to the case of $2 \times 2$ matrix $A$. The related equation (1) is reduced to the form:

$$
\begin{aligned}
& \frac{\partial}{\partial t} u_{1}-\sum_{i} \frac{\partial^{2}}{\partial x_{i}^{2}}\left(A^{11} u_{1}+A^{12} u_{2}\right)=f^{1}, \\
& \frac{\partial}{\partial t} u_{2}-\sum_{i} \frac{\partial^{2}}{\partial x_{i}^{2}}\left(A^{21} u_{1}+A^{22} u_{2}\right)=f^{2}
\end{aligned}
$$

where $A^{11}, A^{12}, A^{21}$ and $A^{22}$ are elements of matrix $A, f^{1}$ and $f^{2}$ are functions of $u_{1}, u_{2}$ to be specified.

First we present all non-equivalent matrices $A$ which have to be considered in the analysis. The ad hoc non-equivalent versions of equation (24) correspond to the following matrices $A$ :

$$
\text { Ia. } A=\left(\begin{array}{cc}
1 & 0 \\
0 & 1
\end{array}\right) ; \quad \text { Ib. } A=\left(\begin{array}{cc}
1 & 0 \\
0 & a
\end{array}\right) ; \quad I I . A=\left(\begin{array}{cc}
b & -c \\
c & b
\end{array}\right) ; \quad \text { III. } A=\left(\begin{array}{cc}
1 & 0 \\
c & 1
\end{array}\right)
$$

where $a, b, c$ and $e$ are arbitrary parameters, $a \neq 0,1$. All $2 \times 2$ matrices can be reduced to one of the forms (25) using linear and scale transformations of the dependent variables. Moreover, without loss of generality it is possible to set $c=1$ and $e=1$.

The analysis of symmetries of systems of nonlinear diffusion equations present in paper [12] was restricted to the case when matrix $A$ had the form $I b$. We consider here the most general case, that is, all possible forms of matrix the $A$ given by relations (25). We present now an outline of the approach used to solve the system (20)-(23)and begin by noticing that: 
- Except the functions $\mu_{a}$ depending on $t, x$ and the constant matrix $A^{-1}$ all quantities in the determining equations belong to one of two classes. Either the quantities depend of $u$ but are independent of $t$, or, alternatively, the quantities depend of $t$ but are independent of $u$. This enables us to separate variable and so decouple the equations.

- To simplify calculations it is convenient to use the equivalence transformations

$$
u^{a} \rightarrow\left(u^{\prime}\right)^{a}=\lambda^{a b} u^{b}+\sigma^{a}, \quad f^{a} \rightarrow\left(f^{\prime}\right)^{a}=\lambda^{a b} f^{b}
$$

where $\lambda^{a b}$ is an invertable constant matrix and $\sigma^{a}$ are constants. Such transformations leave equation (1) form invariant and so make it possible to reduce $A^{-1}$ (and other matrices used in the analysis) to canonical forms (25).

- Many solutions of (20)-(23) are such that $f$ is linear in $u$. Such possibilities are considered separately.

An important a priori simplification of the determining equations can be obtained by considering the compatibility of (20)-(23).

Lemma 1.Let equations (20), (22)be compatible and $f^{k}$ are nonlinear in $u$. Then, up to equivalence transformations (26), the functions $d$ and $\omega_{2}^{k}$ have to satisfy one of the following relations

$$
\dddot{d}=0, \quad \omega_{2}^{1}=0, \quad \dot{\omega}_{2}^{k}=\mu \omega_{2}^{k}-\xi_{2}^{k}+\mu \ddot{d}, \quad \text { or } \quad \dddot{d}=\mu \ddot{d}, \quad \omega_{2}^{1}=\omega_{2}^{2}=0
$$

where $\mu \neq 0, \nu_{1}$ and $\nu_{2}$ are constants, at least one of constants $\nu_{\alpha}$ is nonzero and nontrivial $\omega_{2}^{k}$ are linearly independent of $\ddot{d}$.

The proof of Lemma 1 depends on the analysis of the consequences of differentiating equation (20) first, with respect to $u_{c}$ and then with respect to $u_{a}$ and further considering the conditions for consistency of the resulting system. Details of this analysis are straightforward and are omitted here.

An analogous result, with a similar proof is valid for equation (21) which generates the following restrictions:

$$
\ddot{g}_{a}=0, \quad \omega_{1}^{1 b}=0, \quad \dot{\omega}_{1}^{2 b}=\nu \omega_{1}^{2 b}-\xi_{1}^{2 b}, \quad \text { or } \quad \ddot{g}_{a}=\nu \dot{g}_{a}, \quad \omega_{1}^{1 b}=\omega_{1}^{2 b}=0 .
$$

These conditions are compatible with (20), (21) only when:

$$
\begin{aligned}
& \dot{d}=d_{3} t+d_{4}, \quad g^{a}=g_{3}^{a} t+g_{4}^{a}, \quad \omega_{1}^{2 b}=\nu^{b} \omega_{2}^{2}, \\
& \dot{d}=d_{1} \exp (\nu t)+d_{2}, \quad g^{a}=g_{1}^{a} \exp (\nu t)+g_{2}^{a}, \quad \omega_{1}^{2 b}=\nu^{b} \omega_{2}^{2}
\end{aligned}
$$

where $\nu, \nu^{a}, g_{k}^{a}, d_{k}, k=1 \ldots 4$ are constant.

Let $\mu^{\alpha}$ are trivial then substituting these into (20)-(22) we find that

$$
\begin{gathered}
\left(A^{-1}\right)^{k b} f_{b}=\left(A^{-1}\right)^{a b} u_{b} \frac{\partial f^{k}}{\partial u^{a}}, \quad d_{3} \Leftarrow 0 \quad \text { or } / \text { and } \quad g_{3}^{a} \models 0 \\
{\left[-2\left(d_{3} t+d_{4}\right) \delta^{k b}+C^{k b}\right] f^{b}+\left(\frac{m}{2} d_{3} \delta_{k b}+\dot{C}^{k b}\right) u^{b}=} \\
=\dot{\omega}_{0}^{k}-2 m A^{k 2} \omega_{2}^{2}-\left(\omega_{0}^{a}-C^{a b} u^{b}\right) \frac{\partial f^{k}}{\partial u^{a}}
\end{gathered}
$$




$$
\omega_{2}^{2}\left(\mu \delta_{k 2}-\frac{\partial f^{k}}{\partial u_{2}}\right)=0
$$

or alternatively, the system which includes (33) and the two following equations

$$
\begin{aligned}
& \left(A^{-1}\right)^{k b}\left(f^{b}+\mu u^{b}\right)=\left(A^{-1}\right)^{a b} u_{b} \frac{\partial f^{k}}{\partial u^{a}}, \quad \mu \rightleftharpoons 0, \quad d_{1} \rightleftharpoons 0 \quad \text { or } \quad g_{1} \rightleftharpoons 0, \\
& {\left[\left(2 d_{1} \exp (\mu t)+d_{2}\right) \delta^{k b}+C^{k b}\right] f^{b}+\left(\frac{1}{2} \mu d_{1} \exp (\nu t) \delta^{k b}+\dot{C}^{k b}\right) u^{b}=} \\
& \dot{\omega}_{0}^{k}-2 A^{k 2} \omega_{2}^{2}-\left(\omega_{0}^{a}-C^{a b} u_{b}\right) \frac{\partial f^{k}}{\partial u^{a}} .
\end{aligned}
$$

Thus, the investigation of symmetries for systems of diffusion equations depends on solving the determining equations (31)-(33) and (34), (35), (33). These include arbitrary parameters $d_{k}, \mu$, arbitrary functions of $t$, i.e., $\omega_{0}^{a}, \omega_{2}^{a}$, and an arbitrary matrix $C^{a b}$ which commutes with $A$ and also depend on $t$. The form of $C$ is given as follows.

Lemma 2. A necessary condition of compatibility of system (31)-(33) is that the matrix function $C^{a b}$ has the form

$$
C^{a b}=\phi_{0} F^{a b}+\phi_{1} B^{a b}+\nu\left(\delta^{a b}-F^{a b}\right)
$$

where $F^{a b}, B^{a b}$ are constants and $\phi_{0}, \phi_{1}$ are functions of $t$ satisfying the conditions

$$
\begin{gathered}
F^{a k} B^{k b}-B^{a k} F^{k b}=\alpha B^{a b}+\beta F^{a b} \\
\dot{\phi}_{0}=k_{0} \phi_{0}+k_{1} \phi_{1}+k_{2}, \quad \dot{\phi}_{1}=n_{0} \phi_{0}+n_{1} \phi_{1}+n_{2} .
\end{gathered}
$$

Here $\alpha, \beta, \nu, k_{0}, k_{1}, k_{2}, n_{0}, n_{1}$ and $n_{2}$ are arbitrary constants.

Proof is straightforward but rather cumbersome, so we present its sketch only.

An arbitrary $2 \times 2$ matrix $C$ whose elements are $C^{a b}$ can be expanded as

$$
C=C_{0} I+C_{1} \sigma_{1}+C_{2} \sigma_{2}+C_{3} \sigma_{3}
$$

where

$$
I=\left(\begin{array}{ll}
1 & 0 \\
0 & 1
\end{array}\right), \sigma_{1}=\left(\begin{array}{ll}
0 & 1 \\
1 & 0
\end{array}\right), \sigma_{2}=\left(\begin{array}{cc}
0 & -1 \\
1 & 0
\end{array}\right), \sigma_{3}=\left(\begin{array}{cc}
1 & 0 \\
0 & -1
\end{array}\right),
$$

$C_{0}, C_{1}, C_{2}$ and $C_{3}$ are functions of $t$. Let $k(1 \leq k \leq 4)$ of these functions be linearly independent, then equating in (32) the coefficients for these functions we obtain $k$ systems of equations for $f^{a}$.

Considering the case $k=4$ it is possible to convince ourselves that the related overdetermined systems are incompatible. For $k=3$ the compatibility condition for (32) reduces to the form (36). Then equating coefficients for independent functions $\phi_{0}$ and $\phi_{1}$ in (32) we come to systems of equations for $f^{a}$, which are compatible provided relations (36) and (37) are satisfied.

Substituting (27), (28) into (32) we obtain

$$
\begin{aligned}
& \phi_{0}\left[F^{k b}\left(f^{b}+k_{0} u^{b}\right)-F^{a b} u_{b} \frac{\partial f^{k}}{\partial u_{a}}+n_{0} B^{k b} u^{b}\right]+\phi_{1}\left[B^{k b}\left(f^{b}+n_{1} u^{b}\right)-\right. \\
& \left.-B^{a b} u_{b} \frac{\partial f^{k}}{\partial u^{a}}+k_{1} F^{k b} u^{b}\right]+\nu\left[\left(\delta^{k b}-F^{k b}\right) F^{b}-\left(\delta^{a b}-F^{a b}\right) u_{b} \frac{\partial f^{k}}{\partial u_{a}}\right]+\left(n_{2} F^{k b}\right. \\
& \left.+k_{2} B^{a b}\right) u_{b}-2\left(d_{3} t+d_{4}\right) f^{k}+\frac{1}{2} d_{3} u^{k}=-\dot{\omega}_{0}^{k}+2 m \delta_{k 2} A^{k 2} \omega_{2}^{2}+2 \omega_{0}^{a} \frac{\partial f^{k}}{\partial u^{a}} .
\end{aligned}
$$


This equation has to be imposed together with (31) provided $g_{3}^{a} \Leftarrow 0$ or $d_{3} \Leftarrow 0$. Moreover, different combinations of values of these parameters correspond to different systems of determining equations. We specify the following five cases:

$$
\begin{aligned}
& d_{3}=0, \quad g_{3}^{a}=0, \quad \phi_{0} \equiv 0, \quad \omega_{2}^{2}=\omega_{1}^{2 b}=0, \quad \omega_{0}^{a}=\text { const }, \quad n_{2}=k_{2}=0 \\
& d_{3}=0, \quad g_{3}^{a}=0, \quad \phi_{0} \equiv 0, \quad \omega_{2}^{2}=\omega_{1}^{2 b}=0, \quad \omega_{0}^{a}=\text { const }, n_{2}=k_{2}=0 \\
& d_{3}=0, \quad g_{3}^{a} \neq 0, \quad \omega_{2}^{2}=\omega_{1}^{2 b}=0, \quad \omega_{0}^{a}=\text { const }, \\
& d_{3} \vDash 0, \quad \omega_{2}^{2}=\omega_{1}^{2 b}=0, \quad \omega_{0}^{a}=\text { const }, \\
& \omega_{2}^{2} \neq 0, \quad \omega_{1}^{2 b} \neq 0 .
\end{aligned}
$$

In this way the system of equations (31)-(33) may be solved explicitly using the method of characteristics to determine $f^{k}$ and their corresponding symmetries. A similar approach can be used for the alternative system (34) and (35) and for the case when $\mu^{\alpha}$ are not trivial. In the last case very strong restriction are imposed on $f^{k}$ by relation (23) which has only few solutions.

\section{Non-linearities and symmetries}

We will not give the detailed calculations but present the general solution of relations (20)-(23). In the following tables we present the results of the symmetry analysis for the case where $f^{k}$ is non-linear in $u$.

First we present the list of non-linearities of the most general form which are defined up to arbitrary functions. To make this we specify matrices $B$ which commute with $A$ (25) according to the following categories:

$$
\begin{aligned}
& \text { I. } B=\left(\begin{array}{ll}
1 & 0 \\
0 & d
\end{array}\right), \quad A^{-1}=\left(\begin{array}{cc}
1 & 0 \\
0 & a^{-1}
\end{array}\right) \text { if } d \neq 1 ; A^{-1} \text { is arbitrary if } d=1 ; \\
& \text { II. } B=\left(\begin{array}{cc}
d & -1 \\
1 & d
\end{array}\right), \quad A^{-1}=\frac{1}{b^{2}+c^{2}}\left(\begin{array}{cc}
b & c \\
-c & b
\end{array}\right) \\
& \text { III a. } B=\left(\begin{array}{ll}
0 & 0 \\
1 & 0
\end{array}\right), \quad A^{-1}=\left(\begin{array}{cc}
1 & 0 \\
-c & 1
\end{array}\right) \\
& \text { IIIIb.B }=\left(\begin{array}{ll}
1 & 0 \\
1 & 1
\end{array}\right), \quad A^{-1}=\left(\begin{array}{cc}
1 & 0 \\
-c & 1
\end{array}\right) .
\end{aligned}
$$

Table 2. Non-linearities with arbitrary functions 


\begin{tabular}{|c|c|c|c|c|c|}
\hline No & $\begin{array}{l}\text { Nonlinear } \\
\text { terms }\end{array}$ & $\begin{array}{l}\text { Type of } \\
\text { matrix } \\
B(41)\end{array}$ & $\begin{array}{c}\text { Argu- } \\
\text { ments of } \\
\varphi_{1}, \varphi_{2}\end{array}$ & $\begin{array}{l}\text { Conditions } \\
\text { for para- } \\
\text { meters }\end{array}$ & $\begin{aligned} & \text { Symmetries, } Z_{N} \\
& \text { for } A^{-1} \vDash \kappa B \\
& \& Z_{E} \text { for } A^{-1}=\kappa B \\
&\end{aligned}$ \\
\hline \multirow[t]{4}{*}{1.} & \multirow[t]{4}{*}{$\begin{array}{l}f^{1}=u_{1}^{k+1} \varphi_{1} \\
f^{2}=u_{1}^{k+d} \varphi_{2}\end{array}$} & \multirow[t]{4}{*}{$I$} & \multirow[t]{4}{*}{$\frac{u_{2}}{u_{1}^{d}}$} & $k \neq 0$ & $\begin{array}{l}Z_{N}=Z_{E}= \\
=X_{0}+\nu D_{1}\end{array}$ \\
\hline & & & & $\begin{array}{c}k=0 \\
d \neq 0\end{array}$ & $\begin{array}{c}Z_{N}=X_{0}+\lambda \hat{B} \\
Z_{E}=Z_{N}+\sigma_{a} G_{a}\end{array}$ \\
\hline & & & & $\begin{array}{l}k=d=0 \\
\varphi_{1} \neq \mathrm{const}\end{array}$ & $Z_{N}=X_{0}+\alpha u_{1} \frac{\partial}{\partial u_{1}}$ \\
\hline & & & & $\begin{array}{c}k=d=0 \\
\varphi_{1}=n\end{array}$ & $\begin{array}{c}Z_{N}=X_{0} \\
+\alpha u_{1} \frac{\partial}{\partial u_{1}}+\tilde{\psi}_{n} \frac{\partial}{\partial u_{1}}\end{array}$ \\
\hline 2. & $\begin{array}{c}f^{1}=e^{k \theta}\left(\varphi_{1} u_{2}+\varphi_{2} u_{1}\right) \\
f^{2}=e^{k \theta}\left(\varphi_{2} u_{2-} \varphi_{1} u_{1}\right)\end{array}$ & $I I$ & $R e^{-d \theta}$ & $k \neq 0$ & $\begin{array}{l}Z_{N}=Z_{E}= \\
=X_{0}+\nu D_{1}\end{array}$ \\
\hline \multirow[t]{3}{*}{3.} & \multirow{3}{*}{$\begin{array}{c}f^{1}=\varphi_{1} u_{1}^{k+1} \\
f^{2}=\left(\varphi_{1} \ln u_{1}+\varphi_{2}\right) u_{1}^{k+1}\end{array}$} & \multirow[t]{3}{*}{$I I I b$} & \multirow[t]{3}{*}{$u_{1} e^{\frac{-u_{2}}{u_{1}}}$} & $k \neq 0$ & $Z_{N}=Z_{E}=X_{0}+\nu D_{1}$ \\
\hline & & & & $\begin{array}{c}k=0 \\
\varphi_{1} \neq \text { const }\end{array}$ & $Z_{N}=X_{0}+\nu u_{1} \frac{\partial}{\partial u_{2}}$ \\
\hline & & & & $\begin{array}{c}k=0 \\
\varphi_{1}=n\end{array}$ & $\begin{aligned} Z_{N}= & X_{0}+\nu u_{1} \frac{\partial}{\partial u_{2}} \\
& +\mu \psi_{n} \frac{\partial}{\partial u_{2}}\end{aligned}$ \\
\hline 4. & $\begin{array}{c}f^{1}=e^{k \frac{u_{2}}{u_{1}}} \varphi_{1} u_{1} \\
f^{2}=e^{k \frac{u_{2}}{u_{1}}}\left(\varphi_{1} u_{2}+\varphi_{2}\right)\end{array}$ & III & $u_{1}$ & $k \neq 0$ & $Z_{N}=X_{0}+\nu D_{1}$ \\
\hline 5 . & $\begin{array}{c}f^{1}=u_{1}\left(n \ln u_{1}+\varphi_{1}\right) \\
f^{2}=u_{2}\left(n \ln u_{2}+\varphi_{2}\right)\end{array}$ & $I$ & $\frac{u_{2}}{u_{1}^{d}}$ & $n \neq 0$ & $\begin{array}{c}Z_{N}=X_{0}+\mu e^{n t} \hat{B} \\
Z_{E}=Z_{N}+\nu_{a} \hat{G}_{a}\end{array}$ \\
\hline \multirow[t]{2}{*}{6.} & \multirow{2}{*}{$\begin{array}{c}f^{1}=\varphi_{1} u_{2}+\varphi_{2} u_{1} \\
+\frac{n}{2}\left(\frac{1}{d} \ln R+\theta\right)\left(d u_{1}-u_{2}\right) \\
f^{2}=\varphi_{2} u_{2-} \varphi_{1} u_{1} \\
+\frac{n}{2}\left(\frac{1}{d} \ln R+\theta\right)\left(d u_{2}+u_{1}\right)\end{array}$} & \multirow[t]{2}{*}{$\begin{array}{c}I I \\
d \neq 0\end{array}$} & \multirow[t]{2}{*}{$R e^{-d \theta}$} & $n \neq 0$ & $\begin{array}{c}Z_{N}=X_{0}+\mu e^{n t} \hat{B} \\
Z_{E}=Z_{N}+\nu_{a} \hat{G}_{a}\end{array}$ \\
\hline & & & & $n=0$ & $\begin{array}{c}Z_{N}=X_{0}+\mu \hat{B} \\
Z_{E}=Z_{N}+\lambda_{a} G_{a}\end{array}$ \\
\hline \multirow[t]{2}{*}{7.} & \multirow[t]{2}{*}{$\begin{array}{c}f^{1}=\left(\varphi_{1}-n \theta\right) u_{2}+\varphi_{2} u_{1} \\
f^{2}=\varphi_{2} u_{2}-\varphi_{1} u_{1}\end{array}$} & \multirow[t]{2}{*}{$\begin{array}{c}I I \\
d=0\end{array}$} & \multirow[t]{2}{*}{$R$} & $n \neq 0$ & $\begin{array}{c}Z_{N}=X_{0}+\mu e^{n t} \hat{B} \\
Z_{E}=Z_{N}+\nu_{a} \hat{G}_{a}\end{array}$ \\
\hline & & & & $n=0$ & $\begin{array}{c}Z_{N}=X_{0}+\mu \hat{B} \\
Z_{E}=Z_{N}+\lambda_{a} G_{a}\end{array}$ \\
\hline \multirow[t]{2}{*}{8.} & \multirow{2}{*}{$\begin{array}{l}f^{1}=\varphi_{1} u_{1}+n u_{2} \\
f^{2}=\varphi_{1} u_{2}+u_{1} \varphi_{2} \\
+n u_{2}\left(1+\frac{u_{2}}{u_{1}}\right)\end{array}$} & \multirow[t]{2}{*}{$I I I b$} & \multirow[t]{2}{*}{$\frac{u_{2}}{u_{1}}-\ln u_{1}$} & $n \neq 0$ & $\begin{array}{c}Z_{N}=X_{0}+\mu e^{n t} \hat{B} \\
Z_{E}=Z_{N}+\nu_{a} \hat{G}_{a}\end{array}$ \\
\hline & & & & $n=0$ & $\begin{array}{c}Z_{N}=X_{0}+\mu \hat{B} \\
Z_{E}=Z_{N}+\lambda_{a} G_{a}\end{array}$ \\
\hline \multirow[t]{3}{*}{9.} & \multirow{3}{*}{$\begin{array}{l}f^{1}=\varphi u_{1}-s u_{1} \\
f^{2}=\varphi u_{2}-n u_{1}\end{array}$} & \multirow[t]{3}{*}{$I I I a$} & \multirow[t]{3}{*}{$u_{1}$} & $s=0, n \neq 0$ & $\begin{aligned} Z_{N}= & X_{0}+\mu u_{1} \frac{\partial}{\partial u_{2}} \\
& +\nu Y_{1}\end{aligned}$ \\
\hline & & & & $s \neq 0, n=0$ & $\begin{aligned} Z_{N} & =X_{0}+\nu u_{2} \frac{\partial}{\partial u_{2}} \\
& +\mu e^{s t} u_{1} \frac{\partial}{\partial u_{2}}\end{aligned}$ \\
\hline & & & & $n=s=0$ & $\begin{aligned} Z_{N}= & X_{0}+\nu u_{2} \frac{\partial}{\partial u_{2}} \\
& +\mu u_{1} \frac{\partial}{\partial u_{2}}\end{aligned}$ \\
\hline
\end{tabular}


Table 2. Continued

\begin{tabular}{|c|c|c|c|c|c|}
\hline 10. & $\begin{array}{c}f^{1}=\varphi_{1} u_{1}, \\
f^{2}=\varphi_{1} u_{2}+\varphi_{2} u_{1}+n u_{2}\end{array}$ & $I I I a$ & $u_{1}$ & $\begin{array}{l}\varphi_{1} \neq 0 \\
\varphi_{1}=0\end{array}$ & $\begin{array}{c}Z_{N}=X_{0}+\mu e^{n t} \hat{B} \\
Z_{N}=X_{0}+\mu \hat{B}+\psi_{n} \frac{\partial}{\partial u_{0}}\end{array}$ \\
\hline 11. & $\begin{array}{c}f^{1}=\varphi_{1} u_{1}^{1-k} \\
f^{2}=\varphi_{2} u_{1}^{-k}\end{array}$ & $\begin{array}{c}I \\
d=0\end{array}$ & $\ln u_{1}+n u_{2}$ & $k \neq 0$ & $Z_{N}=X_{0}+\nu D_{2}$ \\
\hline \multirow[t]{2}{*}{12.} & \multirow{2}{*}{$\begin{array}{c}f^{1}=\varphi_{1} u_{1}+\frac{s}{n} u_{1} u_{2} \\
f^{2}=\varphi_{2}+s u_{2}\end{array}$} & \multirow{2}{*}{$\begin{array}{c}I \\
d=0\end{array}$} & \multirow[t]{2}{*}{$u_{2}-n \ln u_{1}$} & $n \neq 0, s=0$ & $Z_{N}=X_{0}+\lambda Y_{2}$ \\
\hline & & & & $s \neq 0, n \neq 0$ & $Z_{N}=X_{0}+\lambda Y_{3}$ \\
\hline \multirow[t]{2}{*}{13.} & \multirow{2}{*}{$\begin{array}{c}f^{1}=e^{k u_{1}} \varphi_{1} \\
f^{2}=e^{k u_{1}}\left(\varphi_{2}-n \varphi_{1} u_{1}\right)\end{array}$} & \multirow[t]{2}{*}{$I I I a$} & \multirow[t]{2}{*}{$n u_{1}^{2}+2 u_{2}$} & $k \neq 0$ & $Z_{N}=X_{0}+\lambda D_{3}$ \\
\hline & & & & $k=0, n \neq 0$ & $Z_{N}=X_{0}+\lambda Y_{4}$ \\
\hline 14. & $\begin{array}{c}f^{1}=n u_{1}+\varphi_{1} \\
f_{2}=k u_{2}+\varphi_{1} u_{1}+\varphi_{2}\end{array}$ & $I I I b$ & $n u_{1}^{2}+2 u_{2}$ & $k \neq 0$ & $\begin{array}{c}Z_{N}=X_{0}+ \\
+\nu e^{n t}\left(u_{1} \frac{\partial}{\partial u_{2}}+\frac{\partial}{\partial u_{1}}\right)\end{array}$ \\
\hline 15. & $\begin{array}{c}f^{1}=n \\
f^{2}=k u_{2}+\varphi\end{array}$ & $I I I a$ & $u_{1}$ & $\varphi \neq$ const & $\begin{aligned} Z_{N} & =X_{0}+\lambda Y_{5} \\
& +\psi_{k} \frac{\partial}{\partial u_{2}}\end{aligned}$ \\
\hline 16 & $\begin{array}{c}f^{1}=(n-r) u_{1}+\varphi_{1} \\
f_{2}=-r u_{2}+\varphi_{2}\end{array}$ & $I I I b$ & $u_{2}$ & & $\begin{array}{c}Z_{N}=X_{0}+ \\
+\nu e^{n t} \psi(x) \frac{\partial}{\partial u_{1}}\end{array}$ \\
\hline 17 & $\begin{array}{c}f^{1}=u_{1} \varphi_{1}+n u_{1} \ln u_{1}, \\
f^{2}=\varphi_{2}\end{array}$ & $\begin{array}{c}I \\
d=0\end{array}$ & $u_{2}$ & $n \neq 0$ & $Z_{N}=X_{0}+\lambda Y_{6}$ \\
\hline 18. & $f^{\alpha}=e^{k u_{1}} \varphi^{\alpha}, \alpha=1,2$ & any & $\omega_{1} u_{2}-\omega_{2} u_{1}$ & $k \vDash 0$ & $Z_{N}=Z_{E}=X_{0}+\nu D_{4}$ \\
\hline \multirow[t]{2}{*}{19.} & \multirow[t]{2}{*}{$\begin{array}{l}f^{1}=\varphi_{1}+s u_{1} \\
f^{2}=\varphi_{2}+s u_{2}\end{array}$} & any & $u_{2}-k u_{1}$ & $\begin{array}{c}k \neq 0 \\
A \neq \varkappa I\end{array}$ & $\begin{array}{c}Z_{N}=Z_{E}=X_{0} \\
+e^{s t} \psi(x)\left(\frac{\partial}{\partial u_{1}}+k \frac{\partial}{\partial u_{2}}\right)\end{array}$ \\
\hline & & $A=\varkappa I$ & $u_{1}$ & $s=0, A=\varkappa I$ & $Z_{N}=X_{0}+\widetilde{\psi_{0}} \frac{\partial}{\partial u_{2}}$ \\
\hline 20 & $f^{1}=\varphi_{1}, f^{2}=\varphi_{2}$ & any & $u_{1}, u_{2}$ & & $Z_{N}=Z_{E}=X_{0}$ \\
\hline
\end{tabular}

Here $R=\sqrt{u_{1}^{2}+u_{2}^{2}}, \theta=\arctan \left(\frac{u_{2}}{u_{1}}\right)$, the Greek letters in the right column denote arbitrary coefficients whilst $D_{\mu}, G_{a}^{i}$ and $\bar{G}_{a}^{i}, X_{A}, Y_{a}, \hat{B}$ are various types of dilatation, Galilei and special transformation generators as follows:

$$
\begin{aligned}
& D_{0}=2 t \frac{\partial}{\partial t}+x_{a} \frac{\partial}{\partial x_{a}}, \quad D_{1}=D_{0}-\frac{2}{k} \hat{B}, \quad D_{2}=D_{0}+\frac{2}{k}\left(\frac{\partial}{\partial u_{2}}+n u_{1} \frac{\partial}{\partial u_{1}}\right), \\
& D_{3}=D_{0}-\frac{2}{k}\left(\frac{\partial}{\partial u_{1}}-n u_{1} \frac{\partial}{\partial u_{2}}\right), \quad D_{4}=D_{0}-\frac{2}{k} \omega_{a} \frac{\partial}{\partial u_{a}}, \\
& G_{a}=t \frac{\partial}{\partial x_{a}}-\frac{1}{2} x_{a}\left(A^{-1}\right)^{n b} u_{b} \frac{\partial}{\partial u_{n}}, \quad \hat{G}_{a}=e^{n t}\left(\frac{\partial}{\partial x_{a}}-\frac{1}{2} n x_{a}\left(A^{-1}\right)^{n b} u_{b} \frac{\partial}{\partial u_{n}}\right), \\
& X_{0}=\alpha \frac{\partial}{\partial t}+\beta_{a} \frac{\partial}{\partial x_{a}}+\nu^{[a, b]} x_{a} \frac{\partial}{\partial x_{b}}, \quad \nu^{[a, b]}=-\nu^{[b, a]}, \\
& Y_{1}=n t u_{1} \frac{\partial}{\partial u_{2}}+u_{2} \frac{\partial}{\partial u_{2}}, \quad Y_{2}=u_{1} \frac{\partial}{\partial u_{1}}+n \frac{\partial}{\partial u_{2}}, \quad Y_{3}=e^{s t}\left(u_{1} \frac{\partial}{\partial u_{1}}+n \frac{\partial}{\partial u_{2}}\right), \\
& Y_{4}=n u_{1} \frac{\partial}{\partial u_{2}}-\frac{\partial}{\partial u_{1}}, Y_{5}=e^{k t}\left(u_{1} \frac{\partial}{\partial u_{2}}+\frac{n x^{2}}{2 m} \frac{\partial}{\partial u_{2}}\right), Y_{6}=e^{n t} u_{1} \frac{\partial}{\partial u_{1}}, \\
& \hat{B}=B^{a b} u_{b} \frac{\partial}{\partial u_{a}}
\end{aligned}
$$

where $B^{a b}$ are elements of the corresponding matrices (41). In addition, $\psi(x)$ and $\widetilde{\psi}_{0}$ are arbitrary solutions of equations $\Delta \psi(x)=0$ and $\left(\frac{\partial}{\partial t}-\Delta\right) \widetilde{\psi}_{0}=0$ respectively were $\Delta$ is the Laplace operator.

In the following Tables 3 and 4 we use triplets of matrices $\left(F, B, A^{-1}\right)$ with $F$ and $B$ forming two-dimensional Lie algebra and commuting with $A(25)$. We classify such 
triplets according to the categories:

$$
\text { I. } \quad F=\left(\begin{array}{ll}
1 & 0 \\
0 & d
\end{array}\right), \quad B=\left(\begin{array}{cc}
0 & 0 \\
0 & 1
\end{array}\right), \quad A^{-1}=\left(\begin{array}{cc}
1 & 0 \\
0 & a^{-1}
\end{array}\right) \text {; }
$$

II $a . \quad F=\left(\begin{array}{cc}1 & 0 \\ 0 & 1\end{array}\right), \quad B=\left(\begin{array}{cc}0 & -1 \\ 1 & 0\end{array}\right), \quad A^{-1}=\frac{1}{b^{2}+c^{2}}\left(\begin{array}{cc}b & c \\ -c & b\end{array}\right)$;

IIb. $\quad F=\left(\begin{array}{cc}d & -1 \\ 1 & d\end{array}\right), \quad B=\left(\begin{array}{ll}1 & 0 \\ 0 & 1\end{array}\right), \quad A^{-1}=\frac{1}{b^{2}+c^{2}}\left(\begin{array}{cc}b & c \\ -c & b\end{array}\right)$

$$
\begin{array}{ll}
\text { IIIa. } F=\left(\begin{array}{ll}
0 & 0 \\
1 & 0
\end{array}\right), \quad B=\left(\begin{array}{ll}
1 & 0 \\
0 & d
\end{array}\right), \quad A^{-1}=\left(\begin{array}{ll}
1 & 0 \\
0 & 1
\end{array}\right) ; \\
\text { IIIb. F }=\left(\begin{array}{ll}
1 & 0 \\
d & 1
\end{array}\right), \quad B=\left(\begin{array}{ll}
0 & 0 \\
1 & 0
\end{array}\right), \quad A^{-1}=\left(\begin{array}{cc}
1 & 0 \\
-c & 1
\end{array}\right)
\end{array}
$$

\begin{tabular}{|c|c|c|c|c|}
\hline No & Non-linear terms & $\begin{array}{l}\text { Conditions } \\
\text { for } \\
\text { parameters } \\
\end{array}$ & $\begin{array}{c}\text { Symmetries, } Z_{N} \\
\text { for, } A^{-1} \neq \kappa F \\
\& Z_{E} \text { for } A^{-1}=\kappa F \\
\end{array}$ & $\begin{array}{c}\text { Matrices }(43) \\
\text { and generator } \\
\text { parameters } \\
\end{array}$ \\
\hline \multirow[t]{5}{*}{1} & $f^{1}=\left(g u_{1}^{q} u_{2}^{r}+n\right) u_{1}$ & $\begin{array}{c}q \neq 0,-1 \\
p \neq 0, n=0 \\
q+r=\frac{4}{m}\end{array}$ & $\begin{array}{l}Z_{N}=X_{0}+\mu \hat{F}+\nu D_{5}, \\
Z_{E}=Z_{N}+\sigma_{a} G_{a}+\lambda \hat{A}\end{array}$ & $\begin{array}{l}I, k=\frac{4}{m} \\
\quad d=-\frac{q}{r}\end{array}$ \\
\hline & \multirow[t]{4}{*}{$f^{2}=\left(p u_{1}^{q} u_{2}^{r}-\frac{q n}{r}\right) u_{2}$} & $\begin{array}{c}q=-1 \\
p=n=0 \\
r=\frac{4+m}{m}\end{array}$ & $\begin{array}{c}Z_{N}=X_{0}+\mu \hat{F}+\nu D_{5} \\
+\widetilde{\psi}_{0} \frac{\partial}{\partial u_{1}} \\
Z_{E}=Z_{N}+\sigma_{a} G_{a}+\lambda \hat{A}\end{array}$ & $\begin{array}{l}I, k=\frac{4}{m} \\
\quad d=\frac{1}{r}\end{array}$ \\
\hline & & $\begin{array}{c}q \neq 0,-1, r \neq 0 \\
q+r \neq 0, \frac{4}{m}\end{array}$ & $\begin{array}{c}Z_{N}=X_{0}+\nu \hat{F}+\mu D_{5} \\
Z_{E}=Z_{N}+\sigma_{a} G_{a}\end{array}$ & $\begin{aligned} I, k & =r+q \\
d & =-\frac{q}{r}\end{aligned}$ \\
\hline & & $\begin{array}{c}p=n=0, g \neq 0 \\
q=-1, r \neq 0,-1\end{array}$ & $\begin{array}{c}Z_{N}=X_{0}+\nu \hat{F} \\
+\mu D_{6}+\tilde{\psi}_{0} \frac{\partial}{\partial u_{1}} \\
Z_{E}=Z_{N}+\sigma_{a} G_{a}\end{array}$ & $\begin{array}{l}I, d=\frac{1}{r} \\
\quad k=r\end{array}$ \\
\hline & & $\begin{array}{c}q=g=0 \\
r \neq 0,-1 ; p \neq 0\end{array}$ & $\begin{array}{c}Z_{N}=X_{0}+\nu \hat{F} \\
+\mu D_{6}+\tilde{\psi}_{n} \frac{\partial}{\partial u_{1}}\end{array}$ & $\begin{array}{c}I, d=0 \\
k=r\end{array}$ \\
\hline \multirow[t]{4}{*}{2} & \multirow{2}{*}{$\begin{array}{c}f^{1}=e^{q \theta} R^{r}\left(g u_{1}\right. \\
\left.-p u_{2}\right)+s u_{2}-l u_{1} \\
f^{2}=e^{q \theta} R^{r}\left(g u_{2}\right. \\
\left.+p u_{1}\right)-s u_{1}-l u_{2}\end{array}$} & $\begin{array}{c}s=l=0 \\
r=\frac{4}{m}\end{array}$ & $\begin{array}{l}Z_{N}=X_{0}+\nu \hat{F}+\mu D_{5} \\
Z_{E}=Z_{N}+\sigma_{a} G_{a}+\lambda \hat{A}\end{array}$ & $\begin{array}{c}I I b, k=\frac{4}{m}, \\
d=-\frac{q}{r}, n=0\end{array}$ \\
\hline & & $\begin{array}{c}r \neq \frac{4}{m}, r \neq 0 \\
s=l=0\end{array}$ & $\begin{array}{c}Z_{N}=X_{0}+\nu \hat{F}+\mu D_{5} \\
Z_{E}=Z_{N}+\sigma_{a} G_{a}\end{array}$ & $\begin{array}{c}I I b, k=r, \\
d=-\frac{q}{r}, n=0\end{array}$ \\
\hline & $R^{2}=u_{1}^{2}+u_{2}^{2}$ & $\begin{array}{c}l=-\frac{s q}{r} \\
s \neq 0, r \neq 0\end{array}$ & $\begin{array}{c}Z_{N}=X_{0}+\nu \hat{F}+\mu D_{6} \\
Z_{E}=Z_{N}+\sigma_{a} G_{a}\end{array}$ & $\begin{array}{c}I I b, k=r \\
n=-s \\
d=-\frac{q}{r}\end{array}$ \\
\hline & $\theta=\arctan \left(\frac{u_{2}}{u_{1}}\right)$ & $\begin{array}{l}s=0, l \neq 0 \\
q \neq 0, r=0\end{array}$ & $\begin{array}{c}Z_{N}=X_{0}+\nu \hat{F}+\mu D_{6} \\
Z_{E}=Z_{N}+\sigma_{a} G_{a}\end{array}$ & $\begin{array}{c}I I a, k=q \\
n=-l\end{array}$ \\
\hline
\end{tabular}

where $a, b, c$ and $d$ are real parameters $\left(a \neq 0, b^{2}+c^{2} \neq 0\right)$.

Table 3. Non-linearities which generate dilatation symmetry 
Table 3. Continued 1

\begin{tabular}{|c|c|c|c|c|}
\hline \multirow[t]{3}{*}{3} & \multirow{3}{*}{$\begin{array}{c}f^{1}=p u_{1}^{r+1} e^{q \frac{u_{2}}{u_{1}}} \\
-s u^{1} \\
f^{2}=e^{q \frac{u_{2}}{u_{1}}}\left(p u_{2}\right. \\
\left.+g u_{1}\right) u_{1}^{r} \\
-s\left(u_{2}-\frac{r}{q} u_{1}\right)\end{array}$} & $\begin{array}{l}r=-q=\frac{4}{m} \\
\quad s=0\end{array}$ & $\begin{array}{l}Z_{N}=X_{0}+\nu \hat{F}+\mu D_{5} \\
Z_{E}=Z_{N}+\alpha_{a} G_{a}+\lambda \hat{A}\end{array}$ & $\begin{array}{c}I I I b, d=1 \\
k=\frac{4}{m}\end{array}$ \\
\hline & & $\begin{array}{l}-q=r \neq \frac{4}{m} \\
s=0, r \neq 0\end{array}$ & $\begin{array}{c}Z_{N}=X_{0}+\nu \hat{F}+\mu D_{5} \\
\quad Z_{E}=Z_{N}+\alpha_{a} G_{a}\end{array}$ & $\begin{array}{c}I I I b, d=1 \\
k=r\end{array}$ \\
\hline & & $q \neq 0, s \neq 0$ & $\begin{array}{c}Z_{N}=X_{0}+\nu \hat{F}+\mu D_{6} \\
Z_{E}=Z_{N}+\alpha_{a} G_{a}\end{array}$ & $\begin{array}{c}I I I b, k=q, \\
n=-s q, \\
d=-\frac{r}{q}\end{array}$ \\
\hline \multirow[t]{4}{*}{4} & \multirow{4}{*}{$\begin{array}{c}f^{1}=p u_{1}^{k+1} \\
f^{2}=u_{1}^{k}\left(p u_{2}+q u_{1}^{d}\right) \\
+\frac{s}{d+k-1} u_{1}\end{array}$} & $\begin{array}{c}d+k \neq 1 \\
k \neq 0, q \neq 0 \\
s=n \neq 0, p \neq 0\end{array}$ & $\begin{aligned} Z_{N} & =X_{0}+\nu \hat{F} \\
& +\mu D_{6}\end{aligned}$ & $I I I a$ \\
\hline & & $\begin{array}{c}k \neq 0, s=0 \\
q=0, p \neq 0\end{array}$ & $\begin{array}{l}Z_{N}=X_{0}+\nu \hat{F} \\
+\mu D_{5}+\lambda u_{2} \frac{\partial}{\partial u_{2}}\end{array}$ & $I I I a, d=0$ \\
\hline & & $\begin{array}{c}p k \neq 0, q=0 \\
d=0 \\
s=n(k-1) \neq 0\end{array}$ & $\begin{array}{l}Z_{N}=X_{0}+\nu \hat{F} \\
\quad+\mu D_{5}+\lambda Y_{1}\end{array}$ & $I I I a, d=0$ \\
\hline & & $\begin{array}{c}d+k \neq 0,1 \\
k \neq 0, q \neq 0 \\
s=n \neq 0, p=0\end{array}$ & $\begin{array}{l}Z_{N}=X_{0}+\nu \hat{F} \\
+\mu D_{6}+\psi_{0} \frac{\partial}{\partial u_{a}}\end{array}$ & $I I I a$ \\
\hline \multirow[t]{2}{*}{5} & \multirow[t]{2}{*}{$\begin{array}{l}f^{1}=p u_{1}^{k+1} \\
f^{2}=p u_{1}^{k} u_{2} \\
-k n u_{1} \ln u_{1}\end{array}$} & $\begin{array}{c}k \neq 0, n \neq 0 \\
p \neq 0\end{array}$ & $Z_{N}=X_{0}+\nu \hat{F}+\mu D_{6}$ & $\begin{array}{c}I I I a, \\
d=1-k\end{array}$ \\
\hline & & $\begin{array}{c}k \neq 0, n \neq 0 \\
p=0\end{array}$ & $\begin{array}{l}Z_{N}=X_{0}+\nu \hat{F} \\
+\mu D_{6}+\psi_{0} \frac{\partial}{\partial u_{2}}\end{array}$ & $\begin{array}{c}I I I a \\
d=1-k\end{array}$ \\
\hline \multirow[t]{2}{*}{6} & \multirow[t]{2}{*}{$\begin{array}{c}f^{1}=q u_{1}^{r+1} e^{k u_{2}}+s u_{1} \\
f^{2}=p u_{1}^{r} e^{k u_{2}}-\frac{s r}{k}\end{array}$} & $\begin{array}{l}r \neq 0,-1 ; k \neq 0 \\
\quad p \neq 0, q \neq 0\end{array}$ & $\begin{aligned} Z_{n}= & X_{0}+\nu D_{7} \\
& +\mu Y_{2}\end{aligned}$ & $\begin{array}{l}I, d=0 \\
n=-\frac{r}{k}\end{array}$ \\
\hline & & $\begin{array}{c}p=0, r=-1 \\
q \neq 0, s=0 \\
k \neq 0 \\
k \neq 0 \\
s=r=0\end{array}$ & $\begin{array}{l}Z_{N}=X_{0}+\nu D_{7} \\
\quad+\mu Y_{2}+\tilde{\psi}_{0} \frac{\partial}{\partial u_{1}} \\
Z_{N}=X_{0}+\nu u_{1} \frac{\partial}{\partial u_{1}} \\
+\mu\left(D_{0}-\frac{2}{k} \frac{\partial}{\partial u_{1}}\right)\end{array}$ & $\begin{array}{c}I, d=0 \\
r=-1 \\
n=\frac{1}{k} \\
I\end{array}$ \\
\hline \multirow[t]{4}{*}{7} & \multirow[t]{4}{*}{$\begin{array}{c}f^{1}=p e^{(n-k) u_{2}}+h u_{2}, \\
f^{2}=g e^{-k u_{2}}+q\end{array}$} & $\begin{array}{c}q=h=0 \\
k \neq 0, n \neq 0\end{array}$ & $\begin{aligned} Z_{N} & =X_{0}+\nu D_{2} \\
& +\tilde{\psi}_{0} \frac{\partial}{\partial u_{1}}\end{aligned}$ & $I, d=0$ \\
\hline & & $\begin{array}{c}q=0 \\
k \neq 0, h \neq 0\end{array}$ & $Z_{N}=X_{0}+\nu D_{8}+\tilde{\psi}_{0} \frac{\partial}{\partial u_{1}}$ & $I, d=0$ \\
\hline & & $\begin{array}{c}p=q=0 \\
h=0, k \neq 0\end{array}$ & $\begin{array}{l}Z_{N}=X_{0}+\nu D_{2} \\
+\sigma u_{1} \frac{\partial}{\partial u_{1}}+\tilde{\psi}_{0} \frac{\partial}{\partial u_{1}}\end{array}$ & $\begin{array}{l}I, d=0 \\
n=0\end{array}$ \\
\hline & & $\begin{aligned} n= & q=g=0 \\
& p \neq 0\end{aligned}$ & $\begin{array}{l}Z_{N}=X_{0}+\nu D_{9} \\
+\mu u_{2} \frac{\partial}{\partial u_{1}}+\tilde{\psi}_{0} \frac{\partial}{\partial u_{1}}\end{array}$ & III \\
\hline
\end{tabular}


Table 3. Continued 2

\begin{tabular}{|c|c|c|c|c|}
\hline \multirow[t]{3}{*}{8} & \multirow[t]{3}{*}{$\begin{array}{c}f^{1}=g e^{u_{2}+k u_{1}} \\
f^{1}=q e^{u_{2}+k u_{1}}+p\end{array}$} & $k \neq 0$ & $\begin{array}{c}Z_{N}=Z_{E} \\
=X_{0}+\nu D_{10} \\
+\psi(x)\left(\frac{\partial}{\partial u_{1}}-k \frac{\partial}{\partial u_{2}}\right)\end{array}$ & $I, a \neq 1$ \\
\hline & & & $\begin{array}{c}Z_{N}=Z_{E} \\
=X_{0}+\nu D_{11} \\
+\psi(x)\left(\frac{\partial}{\partial u_{1}}-k \frac{\partial}{\partial u_{2}}\right)\end{array}$ & $\begin{array}{l}I I a, I I b \\
\quad c \neq 0\end{array}$ \\
\hline & & $k=0$ & $\begin{array}{c}Z_{N}=Z_{E}=X_{0} \\
+\nu D_{12}+\psi(x) \frac{\partial}{\partial u_{1}}\end{array}$ & $\begin{array}{l}I I I b \\
e \neq 0\end{array}$ \\
\hline 9 & $\begin{array}{c}f^{1}=p\left(u_{2}+n u_{1}^{2}\right)^{s+\frac{1}{2}} \\
+\frac{1}{2 n(2 s+1)} \\
f^{2}=-\frac{1}{2 s+1} u_{1} \\
+\left(q-2 n p u_{1}\right) \\
\times\left(u_{2}+n u_{1}^{2}\right)^{s+\frac{1}{2}}\end{array}$ & $\begin{array}{c}s \neq 0,-\frac{1}{2} \\
p \neq 0, n \neq 0\end{array}$ & $Z_{N}=X_{0}+\nu D_{13}$ & $I I I a$ \\
\hline \multirow[t]{3}{*}{10} & \multirow[t]{3}{*}{$\begin{array}{c}f_{1}=g u_{1}^{k+1} \\
f_{2}=\left(p \ln u_{1}+q\right) u_{1}^{k+1}\end{array}$} & $\begin{array}{c}k=-1, q=0 \\
p \neq 0, g \neq 0\end{array}$ & $\begin{array}{c}Z_{N}=Z_{E}=X_{0} \\
+\psi_{0} \frac{\partial}{\partial u_{2}} \\
+\nu\left(D_{5}+2 p t \frac{\partial}{\partial u_{2}}\right)\end{array}$ & $\begin{aligned} I, k & =-1 \\
d & =0\end{aligned}$ \\
\hline & & $\begin{array}{c}k=-1, p \neq 0 \\
q=g=0\end{array}$ & $\begin{array}{c}Z_{N}=Z_{E}=X_{0} \\
+\nu\left(D_{5}+2 p t \frac{\partial}{\partial u_{2}}\right) \\
+\lambda\left(B \widehat{F}+p t \frac{\partial}{\partial u_{2}}\right) \\
+\psi_{0} \frac{\partial}{\partial u_{2}}\end{array}$ & $\begin{aligned} I, k & =-1 \\
d & =0\end{aligned}$ \\
\hline & & $\begin{array}{l}k \neq 0,-1 \\
\quad g=p\end{array}$ & $\begin{array}{c}Z_{N}=Z_{E}=X_{0} \\
+\nu D_{1}+\psi_{0} \frac{\partial}{\partial u_{2}}\end{array}$ & $I I I b, d=1$ \\
\hline \multirow[t]{2}{*}{11} & \multirow[t]{2}{*}{$\begin{array}{c}f^{1}=g\left(u_{2}+s u_{1}\right)^{k+1} \\
f^{2}=q\left(u_{2}+s u_{1}\right)^{k+1} \\
+p\end{array}$} & $\begin{array}{l}k \neq 0,-1 \\
\quad s \neq 0\end{array}$ & $\begin{array}{c}Z_{N}=Z_{E} \\
=X_{0}+\nu D_{14} \\
+\psi(x)\left(\frac{\partial}{\partial u_{1}}-s \frac{\partial}{\partial u_{2}}\right)\end{array}$ & $I, a \neq 1$ \\
\hline & & $\begin{array}{l}k \neq 0,-1 \\
\quad s=0\end{array}$ & $\begin{array}{c}Z_{N}=Z_{E}=X_{0} \\
+\nu D_{15}+\psi(x) \frac{\partial}{\partial u_{1}}\end{array}$ & $\begin{array}{c}I I a, I I b \\
c \neq 0 \\
I I I b, b=1 \\
c \neq 0\end{array}$ \\
\hline \multirow[t]{2}{*}{12} & \multirow[t]{2}{*}{$\begin{array}{c}f^{1}=p \ln \left(u_{2}+s u_{1}\right) \\
f^{2}=q \ln \left(u_{2}+s u_{1)}\right.\end{array}$} & $s \neq 0$ & $\begin{array}{c}Z_{N}=Z_{E} \\
=X_{0}+\nu D_{16} \\
+\psi(x)\left(\frac{\partial}{\partial u_{1}}-s \frac{\partial}{\partial u_{2}}\right)\end{array}$ & $\begin{array}{c}I, a \neq 1 \\
k=-1\end{array}$ \\
\hline & & $s=0$ & $\begin{array}{c}Z_{N}=Z_{E}=X_{0} \\
+\nu D_{17}+\psi(x) \frac{\partial}{\partial u_{1}}\end{array}$ & $\begin{array}{c}I I a, I I b, \\
k=-1, \\
c \neq 0 ; \\
I I I b, k=-1, \\
b=1, \\
c \neq 0\end{array}$ \\
\hline 13 & $\begin{array}{l}f^{1}=g u_{1}^{k+1} \\
f^{2}=q u_{1}^{k+d}\end{array}$ & $g \neq 0, k \neq 0$ & $\begin{array}{c}Z_{N}=Z_{E}=X_{0} \\
+\nu\left(D_{0}-\frac{2}{k} \widehat{F}\right)+\psi_{0} \frac{\partial}{\partial u_{2}}\end{array}$ & $I$ \\
\hline
\end{tabular}


Table 4. Further non-linearities with arbitrary parameters

\begin{tabular}{|c|c|c|c|c|}
\hline No & $\begin{array}{l}\text { Non-linear } \\
\text { terms }\end{array}$ & $\begin{array}{c}\text { Matrix } \\
\text { Class } \\
(43)\end{array}$ & $\begin{array}{l}\text { Conditions } \\
\text { for parameters } \\
\text { and matrices } \mathcal{F}_{a}\end{array}$ & $\begin{array}{c}\text { Symmetries, } \\
Z_{N} \text { for } A^{-1} \neq \kappa \mathcal{F}_{a} \\
\& Z_{E} \text { for } A^{-1}=\kappa \mathcal{F}_{1} \\
\& \tilde{Z}_{E} \text { for } A^{-1}=\kappa \mathcal{F}_{2}\end{array}$ \\
\hline \multirow[t]{12}{*}{1} & \multirow{12}{*}{$\begin{array}{c}f^{1}= \\
\left(k_{0} \ln u_{1}\right. \\
+k_{1} \ln u_{2} \\
+q) u_{1} \\
f^{2}= \\
\left(n_{0} \ln u_{1}\right. \\
+n_{1} \ln u_{2}\end{array}$} & \multirow[t]{12}{*}{$\begin{array}{l}I, \\
d=0\end{array}$} & $\begin{array}{c}\delta>0, \Delta \neq 0, k_{1} \neq 0 \\
\mathcal{F}_{1}=k_{1} F+\left(n_{+}-k_{0}\right) B \\
\mathcal{F}_{2}=k_{1} F+\left(n_{-}-k_{0}\right) B \\
n_{ \pm}=\frac{k_{0}+n_{1}}{2} \pm \delta\end{array}$ & $\begin{array}{c}Z_{N}=X_{0}+\lambda e^{n_{+} t} \hat{\mathcal{F}}_{1}+\nu e^{n_{-} t} \hat{\mathcal{F}}_{2} \\
Z_{E}=Z_{N}+\sigma_{a} \hat{G}_{a}\left(n=n_{+}\right) \\
\tilde{Z}_{E}=Z_{N}+\sigma_{a} \hat{G}_{a}\left(n=n_{-}\right)\end{array}$ \\
\hline & & & $k_{1}=n_{0}=0, k_{0} n_{1} \neq 0$ & $\begin{aligned} Z_{N}= & X_{0}+\nu e^{k_{o} t} u_{1} \frac{\partial}{\partial u_{1}} \\
& +\mu e^{n_{1} t} u_{2} \frac{\partial}{\partial u_{2}}\end{aligned}$ \\
\hline & & & $k_{1}=n_{0}=n_{1}=0$ & $Z_{N}=X_{0}+\psi_{p} \frac{\partial}{\partial u_{2}}+\nu e^{k_{o} t} u_{1} \frac{\partial}{\partial u_{1}}$ \\
\hline & & & $\begin{array}{c}\Delta=0, k_{1} \neq 0 \\
k_{0}+n_{1}=n \neq 0 \\
\mathcal{F}_{1}=k_{1} F+n_{1} B \\
\mathcal{F}_{2}=k_{0} B-k_{1} F\end{array}$ & $\begin{array}{c}Z_{N}=X_{0} \\
+\lambda e^{\left(n_{1}+k_{0}\right) t} \hat{\mathcal{F}}_{1}+\nu \hat{\mathcal{F}}_{2} \\
Z_{E}=Z_{N}+\sigma_{a} \hat{G}_{a}\left(n_{1} \neq 0\right) \\
\hat{Z}_{E}=Z_{N}+\sigma_{a} G_{a}\left(k_{0} \neq 0\right)\end{array}$ \\
\hline & & & $\begin{array}{c}k_{0}=n_{1}=n \\
k_{1}=n_{0}=0 \\
\mathcal{F}_{1}=\mu F+\nu B, \mu \nu \neq 0\end{array}$ & $\begin{array}{c}Z_{N}=X_{0}+\alpha e^{n t} u_{1} \frac{\partial}{\partial u_{1}} \\
+\lambda e^{n t} u_{2} \frac{\partial}{\partial u_{2}} \\
\tilde{Z}_{E}=Z_{N}+\sigma_{a} \hat{G}_{a}\end{array}$ \\
\hline & & & $\begin{array}{c}k_{0}=n_{1}=n \\
n_{0}=0, k_{1} \neq 0\end{array}$ & $\begin{array}{c}Z_{N}=X_{0}+\lambda e^{n t} u_{1} \frac{\partial}{\partial u_{1}} \\
+\nu e^{n t}\left(k_{1} t u_{1} \frac{\partial}{\partial u_{1}}+u_{2} \frac{\partial}{\partial u_{2}}\right)\end{array}$ \\
\hline & & & $\begin{array}{c}\delta=0, \Delta \neq 0, k_{0}+n_{1}=2 n \\
\mathcal{F}_{1}=2 k_{1} F+\left(n_{1}-k_{0}\right) B, \\
\mathcal{F}_{2}=t \mathcal{F}_{1}+2 B\end{array}$ & $\begin{array}{c}Z_{N}=X_{0}+\nu X_{1}+\mu X_{2} \\
Z_{E}=Z_{N}+\sigma_{a} \hat{G}_{a}\end{array}$ \\
\hline & & & $\begin{array}{c}\delta=\Delta=0 \\
n_{0} k_{1} \neq 0 \\
\mathcal{F}_{1}=k_{1} F-k_{0} B\end{array}$ & $\begin{array}{c}Z_{N}=X_{0}+\nu\left[k_{1} t u_{1} \frac{\partial}{\partial u_{1}}\right. \\
\left.+\left(1-k_{0} t\right) u_{2} \frac{\partial}{\partial u_{2}}\right] \\
+\mu\left(k_{1} u_{1} \frac{\partial}{\partial u_{1}}-k_{0} u_{2} \frac{\partial}{\partial u_{2}}\right) \\
Z_{E}=Z_{N}+\sigma_{a} G_{a}\end{array}$ \\
\hline & & & $\begin{array}{c}k_{1}=n_{1}=0, q=p \\
k_{0}=n_{0}=n, a=1 \\
\mathcal{F}_{1}=F+B\end{array}$ & $\begin{array}{c}Z_{N}=X_{0}+\nu u_{2} \frac{\partial}{\partial u_{2}}+\mu u_{1} \frac{\partial}{\partial u_{2}} \\
+\lambda e^{n t}\left(u_{1} \frac{\partial}{\partial u_{1}}+u_{2} \frac{\partial}{\partial u_{2}}\right) \\
Z_{E}=Z_{N}+\sigma_{a} \tilde{G}_{a}\end{array}$ \\
\hline & & & $\begin{array}{l}k_{1}=n_{1}=0, p=0 \\
k_{0}=n_{0}=n, q \neq 0 \\
a=1, \mathcal{F}_{1}=F+B\end{array}$ & $\begin{array}{c}Z_{N}=X_{0}+\nu u_{2} \frac{\partial}{\partial u_{2}}+\mu e^{p t} u_{1} \frac{\partial}{\partial u_{2}} \\
+\lambda e^{n t}\left(u_{1} \frac{\partial}{\partial u_{1}}+u_{2} \frac{\partial}{\partial u_{2}}\right) \\
Z_{E}=Z_{N}+\sigma_{a} \tilde{G}_{a}\end{array}$ \\
\hline & & & $\begin{array}{c}k_{0}=k_{1}=n_{1}=0 \\
n_{0} \neq 0\end{array}$ & $\begin{array}{c}Z_{N}=X_{0}+\nu u_{2} \frac{\partial}{\partial u_{2}} \\
+\mu\left(u_{1} \frac{\partial}{\partial u_{1}}+n_{0} t u_{2} \frac{\partial}{\partial u_{2}}\right)\end{array}$ \\
\hline & & & $\delta=-\omega^{2}, 2 n=k_{0}+n_{1}$ & $Z_{N}=X_{0}+\nu X_{3}+\mu X_{4}$ \\
\hline
\end{tabular}


Table 4. Continued 1

\begin{tabular}{|c|c|c|c|c|}
\hline \multirow[t]{7}{*}{2} & \multirow[t]{7}{*}{$\begin{array}{l}f^{1}=q u_{1} \\
f^{2}=n u_{2}+p u_{1}^{d} \\
\quad+s u_{1}+k\end{array}$} & \multirow[t]{6}{*}{$I I I a$} & $\begin{array}{c}d \neq 0,1,2 ; k=0 \\
s=0, n \neq q, \mathcal{F}_{1}=B\end{array}$ & $\begin{array}{c}Z_{N}=X_{0}+\nu \hat{B}+\psi_{n} \frac{\partial}{\partial u_{2}} \\
Z_{E}=Z_{N}+\sigma_{a} G_{a}\end{array}$ \\
\hline & & & $\begin{array}{c}d \neq 0,2 ; n=0 \\
s=0 \\
\mathcal{F}_{1}=B\end{array}$ & $\begin{array}{l}Z_{N}=X_{0}+\psi_{0} \frac{\partial}{\partial u_{2}} \\
+\nu\left(\hat{B}-d k t \frac{\partial}{\partial u_{2}}\right) \\
Z_{E}=Z_{N}+\sigma_{a} G_{a}\end{array}$ \\
\hline & & & $\begin{array}{c}d \neq 0,1,2 ; k=0 \\
s=\frac{1}{1-d}, \mathcal{F}_{1}=B\end{array}$ & $\begin{array}{c}Z_{N}=X_{0}+\nu \hat{F} \\
+\mu \hat{B}+\psi_{q} \frac{\partial}{\partial u_{2}} \\
Z_{E}=Z_{N}+\sigma_{a} G_{a}\end{array}$ \\
\hline & & & $d=2, k=0, s \neq 0$ & $Z_{N}=X_{0}+\mu Y_{7}+\psi_{n} \frac{\partial}{\partial u_{2}}$ \\
\hline & & & $\begin{array}{c}n=2(q+p) \\
d=2, k=s=0 \\
\mathcal{F}_{1}=B\end{array}$ & $\begin{array}{c}Z_{N}=X_{0}+\nu Y_{8} \\
+\mu \hat{B}+\psi_{0} \frac{\partial}{\partial u_{2}} \\
Z_{E}=Z_{N}+\sigma_{a} G_{a}\end{array}$ \\
\hline & & & $\begin{array}{c}d=2, p=-q \\
n=s=0\end{array}$ & $\begin{array}{l}Z_{N}=X_{0}+\nu Y_{8}+\psi_{0} \frac{\partial}{\partial u_{2}} \\
\quad+\mu\left(\hat{B}-2 k t \frac{\partial}{\partial u_{2}}\right)\end{array}$ \\
\hline & & $I I I b$ & $\begin{array}{c}k=s=0 \\
d=2, \mathcal{F}_{1}=B\end{array}$ & $\begin{array}{c}Z_{N}=X_{0}+\nu \hat{B} \\
+\mu e^{q t} u_{1} \frac{\partial}{\partial u_{2}}+\psi_{0} \frac{\partial}{\partial u_{2}} \\
Z_{E}=Z_{N}+\sigma_{a} G_{a}\end{array}$ \\
\hline \multirow[t]{5}{*}{3} & \multirow[t]{5}{*}{$\begin{array}{c}f^{1}=k u_{1} \ln u_{1}+p u_{1} \\
f^{2}=b u_{2}+n \ln u_{1}\end{array}$} & \multirow[t]{5}{*}{$I$} & $\begin{array}{c}b=k=q=0 \\
p \neq 0\end{array}$ & $\begin{aligned} Z_{N} & =X_{0}+\nu Y_{9} \\
& +\psi_{0} \frac{\partial}{\partial u_{2}}\end{aligned}$ \\
\hline & & & $q=p=0, b=k$ & $Z_{N}=X_{0}+\nu Y_{10}+\psi_{b} \frac{\partial}{\partial u_{2}}$ \\
\hline & & & $\begin{array}{c}q=p=0 \\
b \neq k, b \neq 0\end{array}$ & $\begin{aligned} Z_{N} & =X_{0}+\nu Y_{11} \\
& +\psi_{b} \frac{\partial}{\partial u_{2}}\end{aligned}$ \\
\hline & & & $k=0, n b \neq 0$ & $\begin{array}{l}Z_{N}=X_{0}+\nu e^{b t} \frac{\partial}{\partial u_{2}} \\
+\mu\left(b u_{1} \frac{\partial}{\partial u_{1}}-n \frac{\partial}{\partial u_{2}}\right)\end{array}$ \\
\hline & & & $\begin{array}{c}b=p=0 \\
k \neq o, n \neq 0\end{array}$ & $\begin{array}{c}Z_{N}=X_{0}+\psi_{0} \frac{\partial}{\partial u_{2}} \\
+\mu e^{k t}\left(k u_{1} \frac{\partial}{\partial u_{1}}+n \frac{\partial}{\partial u_{2}}\right)\end{array}$ \\
\hline \multirow[t]{3}{*}{4} & \multirow{3}{*}{$\begin{array}{c}f^{1}=k_{0} u_{1} \ln u_{1}+k_{1} u_{1} u_{2} \\
f^{2}=n_{0} \ln u_{1}+n_{1} u_{2}\end{array}$} & \multirow{3}{*}{$\begin{array}{c}I \\
d=0\end{array}$} & $k_{1} \neq 0, \delta=0$ & $\begin{array}{c}Z_{N}=X_{0}+\nu Y_{12} \\
+\mu\left(t Y_{12}+e^{\frac{k_{0}+n_{1}}{2} t} t \frac{\partial}{\partial u_{2}}\right)\end{array}$ \\
\hline & & & $k_{1} \neq 0, \delta>0$ & $Z_{N}=X_{0}+\nu Y_{13}^{+}+\mu Y_{13}^{-}$ \\
\hline & & & $k_{1} \neq 0, \delta=-\omega^{2}<0$ & $Z_{N}=X_{0}+\nu Y_{14}+\mu Y_{15}$ \\
\hline 5 & $\begin{array}{c}f^{1}=p u_{2}^{k}+q u_{1} \\
f^{2}=g u_{2}\end{array}$ & $\begin{array}{c}I, \\
d=\frac{1}{k}\end{array}$ & $\begin{array}{c}p \neq 0, k g \neq q \\
k \neq 0,1 ; \mathcal{F}_{1}=F\end{array}$ & $\begin{array}{c}Z_{N}=X_{0}+\nu \hat{F}+\tilde{\psi}_{q} \frac{\partial}{\partial u_{1}} \\
Z_{E}=Z_{N}+\sigma_{a} G_{a}\end{array}$ \\
\hline 6 & $\begin{array}{c}f^{1}=n u_{1} \ln u_{1} \\
f^{2}=n u_{2} \ln u_{1}+p u_{1}\end{array}$ & $\begin{array}{c}I \\
d=0 \\
a=1\end{array}$ & $\begin{array}{c}n \neq 0, p \neq 0 \\
\mathcal{F}_{1}=B+F\end{array}$ & $\begin{array}{c}Z_{N}=X_{0}+\mu u_{1} \frac{\partial}{\partial u_{2}} \\
+\nu\left(\hat{B}-p t u_{1} \frac{\partial}{\partial u_{2}}\right) \\
+\lambda e^{n t}\left(u_{1} \frac{\partial}{\partial u_{1}}+u_{2} \frac{\partial}{\partial u_{2}}\right) \\
Z_{E}=Z_{N}+\sigma_{a} \tilde{G}_{a}\end{array}$ \\
\hline 7 & $\begin{array}{l}f^{1}=p u_{1}^{k+1} \\
f^{2}=p u_{1}^{k} u_{2}+s u_{2}\end{array}$ & $\begin{array}{l}I I I a \\
d=0\end{array}$ & $k \neq 0, s \neq 0$ & $\begin{array}{l}Z_{N}=X_{0}+\nu u_{2} \frac{\partial}{\partial u_{2}} \\
+\mu e^{s t} u_{1} \frac{\partial}{\partial u_{2}}\end{array}$ \\
\hline
\end{tabular}


Table 4.Continued 2

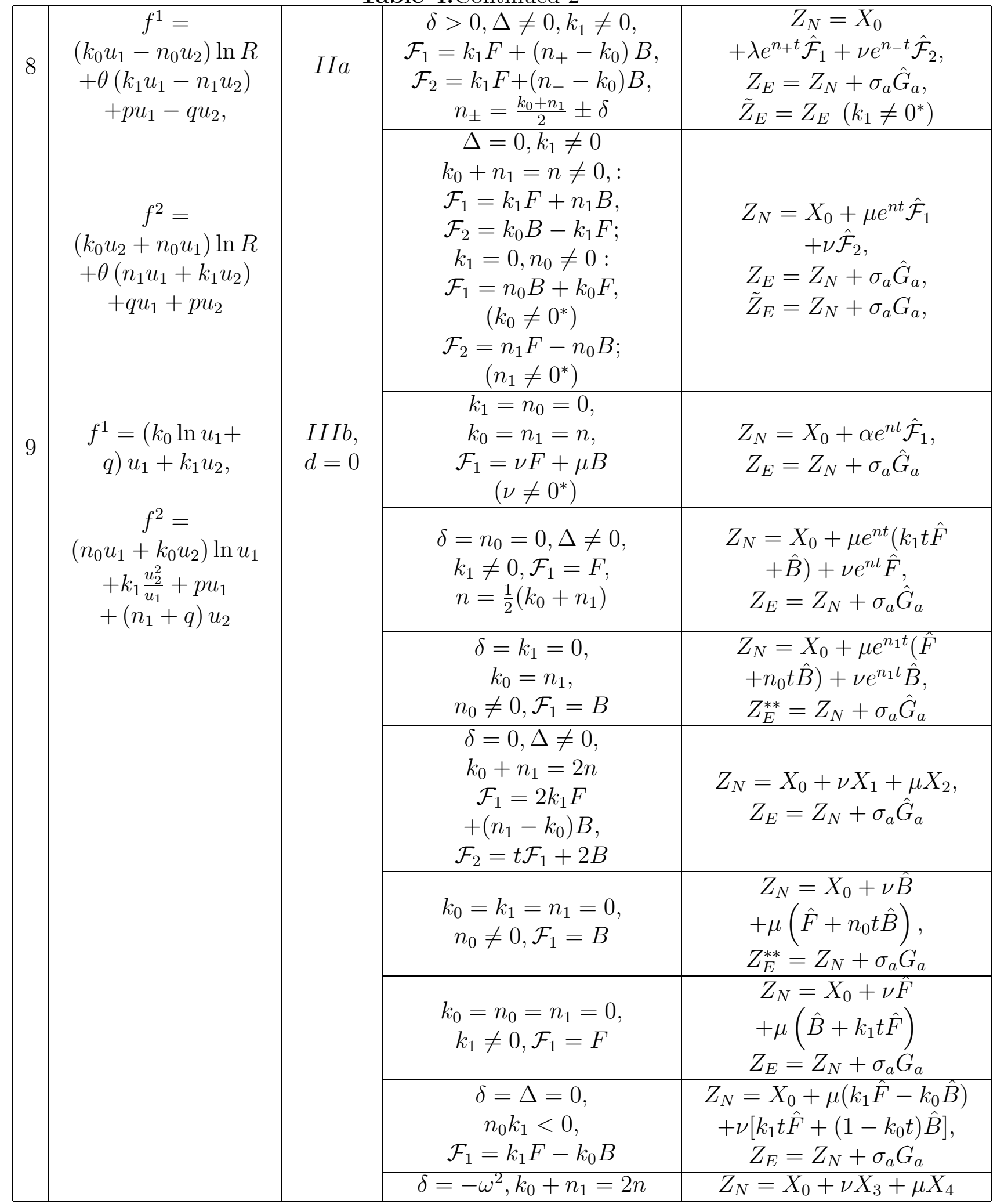


In Table 4 columns 4 and 5 refer to the both non-linearities specified as Cases 8 and 9. However, the conditions marked by ${ }^{*}$ are related to Case 8 only and the symmetries marked by ${ }^{* *}$ correspond only to Case 9 . In addition, $\kappa=$ constant, $\Delta=k_{0} n_{1}-n_{0} k_{1}$, $\delta=\frac{1}{4}\left(k_{0}-n_{1}\right)^{2}+k_{1} n_{0}, \hat{\mathcal{F}}_{\alpha}=\mathcal{F}_{\alpha}^{a b} u_{b} \frac{\partial}{\partial u_{a}}, \hat{F}=F^{a b} u_{b} \frac{\partial}{\partial u_{a}}$ and:

$$
\left(\frac{\partial}{\partial t}-A \sum_{i=1}^{m} \frac{\partial^{2}}{\partial x_{i}^{2}}\right)\left(\begin{array}{c}
0 \\
\psi_{n}
\end{array}\right)=n\left(\begin{array}{c}
0 \\
\psi_{n}
\end{array}\right), \quad\left(\frac{\partial}{\partial t}-A \sum_{i=1}^{m} \frac{\partial^{2}}{\partial x_{i}^{2}}\right)\left(\begin{array}{c}
\tilde{\psi}_{n} \\
0
\end{array}\right)=n\left(\begin{array}{c}
\tilde{\psi}_{n} \\
0
\end{array}\right)
$$

and $n$ is an arbitrary parameter (including the case $n=0$ ). The generators $D_{\mu}, \hat{A}, G_{a}, \hat{G}_{a}, X_{\nu}, Y_{s}$ when not specified in (42) are given by:

$$
\begin{aligned}
& \hat{A}=t^{2} \frac{\partial}{\partial t}+t x_{a} \frac{\partial}{\partial x_{a}}-\frac{1}{4} x^{2}\left(A^{-1}\right)^{a b} u_{b} \frac{\partial}{\partial u_{a}}-\frac{m}{2} t\left(u_{1} \frac{\partial}{\partial u_{1}}+u_{2} \frac{\partial}{\partial u_{2}}\right)+n t^{2} \hat{F}, \\
& D_{5}=D_{0}-\frac{2}{k}\left(u_{1} \frac{\partial}{\partial u_{1}}+u_{2} \frac{\partial}{\partial u_{2}}\right), \quad D_{6}=D_{0}+2 \operatorname{tn} \hat{F}-\frac{2}{k} \hat{B}, \\
& D_{7}=D_{0}+2\left(s t-\frac{1}{r}\right) u_{1} \frac{\partial}{\partial u_{1}}-\frac{2 s r t}{k} \frac{\partial}{\partial u_{2}}, \quad D_{8}=D_{0}+\frac{2 n}{k} u_{1} \frac{\partial}{\partial u_{1}}+\frac{2 h t}{k} \frac{\partial}{\partial u_{1}}+\frac{2}{k} \frac{\partial}{\partial u_{2}}, \\
& D_{9}=D_{0}-2 h t u_{2} \frac{\partial}{\partial u_{1}}-\frac{2}{k} \frac{\partial}{\partial u_{2}} \text {, } \\
& D_{10}=D_{0}-2 \frac{\partial}{\partial u_{2}}-\frac{p}{k(1-a)}\left(\frac{x^{2}}{m}+2 t\right)\left(\frac{\partial}{\partial u_{1}}-k \frac{\partial}{\partial u_{2}}\right) \text {, } \\
& D_{11}=D_{0}-2 \frac{\partial}{\partial u_{2}}-\frac{p}{c\left(1+k^{2}\right)}\left(\frac{x^{2}}{m}+2 t(c k+b)\right)\left(\frac{\partial}{\partial u_{1}}-k \frac{\partial}{\partial u_{2}}\right) \text {, } \\
& D_{12}=D_{0}-2 \frac{\partial}{\partial u_{2}}-\frac{p}{e}\left(\frac{x^{2}}{m}+2 t\right) \frac{\partial}{\partial u_{1}} \text {, } \\
& D_{13}=D_{0}-\frac{1}{s}\left(u_{1} \frac{\partial}{\partial u_{1}}+2 u_{2} \frac{\partial}{\partial u_{2}}\right)+\frac{t}{2 s n} \frac{\partial}{\partial u_{1}}-\frac{t}{s} u_{1} \frac{\partial}{\partial u_{2}}, \\
& D_{14}=D_{5}+\frac{p(k+1)}{k s(a-1)}\left(2 t+\frac{x^{2}}{m}\right)\left(\frac{\partial}{\partial u_{1}}-s \frac{\partial}{\partial u_{2}}\right) \text {, } \\
& D_{15}=D_{5}-\frac{p(k+1)}{k c}\left(2 t b+\frac{x^{2}}{m}\right) \frac{\partial}{\partial u_{1}} \text {, } \\
& D_{16}=D_{5}+\frac{1}{s(a-1)}\left[2 t(q+p s(a-1))+q \frac{x^{2}}{m}\right]\left(\frac{\partial}{\partial u_{1}}-s \frac{\partial}{\partial u_{2}}\right) \text {, } \\
& D_{17}=D_{0}+2\left(u_{1} \frac{\partial}{\partial u_{1}}+u_{2} \frac{\partial}{\partial u_{2}}\right)-\frac{1}{c}\left(2 t(b q-p c)+q \frac{x^{2}}{m}\right) \frac{\partial}{\partial u_{1}}, \\
& X_{1}=\exp (n t)\left(2 k_{1} \hat{F}+\left(n_{1}-k_{0}\right) \hat{B}\right), \quad X_{2}=t X_{1}+2 \exp (n t) \hat{B}, \\
& X_{3}=\exp (n t)\left[k_{1} \cos (\omega t) \hat{F}+\left(\left(\lambda_{0}-k_{0}\right) \cos (\omega t)-\omega \sin (\omega t)\right) \hat{B}\right] \text {, } \\
& X_{4}=\exp (n t)\left[k_{1} \sin (\omega t) \hat{F}+\left(\left(\lambda_{0}-k_{0}\right) \sin (\omega t)+\omega \cos (\omega t)\right) \hat{B}\right] \text {, } \\
& Y_{7}=\exp (n t)\left(u_{1} \frac{\partial}{\partial u_{2}}-\frac{q}{2 p}\left(\frac{s x^{2}}{2 m} \frac{\partial}{\partial u_{2}}-\frac{\partial}{\partial u_{1}}\right)\right), \quad Y_{8}=\exp (n t)\left(u_{1} \frac{\partial}{\partial u_{2}}-\frac{\partial}{\partial u_{1}}\right), \\
& Y_{9}=u_{1} \frac{\partial}{\partial u_{1}}+n t \frac{\partial}{\partial u_{2}}, \quad Y_{10}=\exp (k t)\left(u_{1} \frac{\partial}{\partial u_{1}}+n t \frac{\partial}{\partial u_{2}}\right) \\
& Y_{11}=\exp (k t) u_{1} \frac{\partial}{\partial u_{1}}+\frac{n}{k-b} \frac{\partial}{\partial u_{2}}, \quad Y_{12}=\exp \left(\lambda_{0} t\right)\left(k_{1} u_{1} \frac{\partial}{\partial u_{1}}+\frac{n_{1}-k_{0}}{2} \frac{\partial}{\partial u_{2}}\right), \\
& Y_{13}^{ \pm}=\exp \left(\lambda_{ \pm} t\right)\left(k_{1} u_{1} \frac{\partial}{\partial u_{1}}+\left(\lambda_{ \pm}-k_{0}\right) \frac{\partial}{\partial u_{2}}\right) \\
& \lambda_{ \pm}=\frac{1}{2}\left(k_{0}+n_{1}\right) \pm \sqrt{\delta}, \lambda_{0}=\frac{1}{2}\left(k_{0}+n_{1}\right) \text {, } \\
& Y_{14}=\exp \left(\lambda_{0} t\right)\left[k_{1} \cos (\omega t) u_{1} \frac{\partial}{\partial u_{1}}+\left(\left(\lambda_{0}-k_{0}\right) \cos (\omega t)-\omega \sin (\omega t)\right) \frac{\partial}{\partial u_{2}}\right] \text {, } \\
& Y_{15}=\exp \left(\lambda_{0} t\right)\left[k_{1} \sin (\omega t) u_{1} \frac{\partial}{\partial u_{1}}+\left(\left(\lambda_{0}-k_{0}\right) \sin (\omega t)+\omega \cos (\omega t)\right) \frac{\partial}{\partial u_{2}}\right] \text {. }
\end{aligned}
$$

\section{$5 \quad$ Linear Systems}

Consider now the linear case when $f^{1}$ and $f^{2}$ have the form, $f^{a}=\Lambda^{a b} u_{b}+\lambda_{a}$. In contrast to the one-dimensional cases we find non trivial possibilities corresponding to the non- 
commutation of the matrices $A$ and $\Lambda$ where we specify $A$ in formulae (25).

Table 5: Symmetries for linear systems

\begin{tabular}{|c|c|c|c|c|}
\hline No & $\begin{array}{l}\text { Form of } \\
f^{1} \text { and } f^{2}\end{array}$ & $\begin{array}{l}\text { Type } \\
\text { of } A(25)\end{array}$ & Conditions & Symmetries \\
\hline \multirow[t]{4}{*}{1} & \multirow[t]{4}{*}{$f^{1}=f^{2}=0$} & $I a$ & & $\begin{array}{c}X_{5}+\lambda u_{1} \frac{\partial}{\partial u_{2}} \\
+\nu u_{2} \frac{\partial}{\partial u_{0}}+\mu u_{2} \frac{\partial}{\partial u_{1}}\end{array}$ \\
\hline & & $I b$ & & $X_{5}+\mu u_{2} \frac{\partial}{\partial u_{2}}$ \\
\hline & & $I I$ & & $X_{5}+\lambda\left(u_{1} \frac{\partial}{\partial u_{2}}-u_{2} \frac{\partial}{\partial u_{1}}\right)$ \\
\hline & & $I I I$ & & $X_{5}+\lambda u_{1} \frac{\partial}{\partial u_{2}}$ \\
\hline 2 & $\begin{array}{l}f^{1}=u_{2} \\
f^{2}=0\end{array}$ & $I b$ & & $\begin{array}{l}X_{0}+\psi_{\Lambda}^{a} \frac{\partial}{\partial u_{a}} \\
+\alpha\left(D_{0}+2 u_{1} \frac{\partial}{\partial u_{1}}\right) \\
+\lambda\left(u_{1} \frac{\partial}{\partial u_{1}}+u_{2} \frac{\partial}{\partial u_{2}}\right)\end{array}$ \\
\hline 3 & $\begin{array}{l}f^{1}=a u_{1}+b u_{2}, \\
f^{2}=c u_{1}+d u_{2}\end{array}$ & $I b$ & $\delta>0$ & $\begin{array}{l}X_{0}+\psi_{\Lambda}^{a} \frac{\partial}{\partial u_{a}} \\
+\nu\left(u_{1} \frac{\partial}{\partial u_{1}}+u_{2} \frac{\partial}{\partial u_{2}}\right)\end{array}$ \\
\hline 4 & $\begin{array}{l}f^{1}=a u_{1}+b u_{2}, \\
f^{2}=c u_{1}+d u_{2}\end{array}$ & $I I$ & $(a-d)^{2}+(b+c)^{2} \neq 0$ & $\begin{array}{l}X_{0}+\psi_{\Lambda}^{a} \frac{\partial}{\partial u_{a}} \\
+\nu\left(u_{1} \frac{\partial}{\partial u_{1}}+u_{2} \frac{\partial}{\partial u_{2}}\right)\end{array}$ \\
\hline 5 & $\begin{array}{l}f^{1}=a u_{1}+b u_{2}, \\
f^{2}=c u_{1}+d u_{2}\end{array}$ & $I I I$ & $b^{2}+(a-d)^{2} \neq 0$ & $\begin{array}{l}X_{0}+\psi_{\Lambda}^{a} \frac{\partial}{\partial u_{a}} \\
+\nu\left(u_{1} \frac{\partial}{\partial u_{1}}+u_{2} \frac{\partial}{\partial u_{2}}\right)\end{array}$ \\
\hline
\end{tabular}

In this table the following notation has been employed:

$$
\begin{aligned}
& \delta=b c-\frac{1}{4}(a-d)^{2} \\
& X_{5}=X_{0}+\nu\left(u_{1} \frac{\partial}{\partial u_{1}}+u_{2} \frac{\partial}{\partial u_{2}}\right)+\lambda D_{0}+\psi_{\Lambda}^{a} \frac{\partial}{\partial u_{a}} \\
& +\mu\left(t^{2} \frac{\partial}{\partial t}+t x_{a} \frac{\partial}{\partial x_{a}}-\frac{1}{4} x^{2}\left(A^{-1}\right)^{a b} u_{b} \frac{\partial}{\partial u_{a}}-\frac{m}{2} t\left(u_{1} \frac{\partial}{\partial u_{1}}+u_{2} \frac{\partial}{\partial u_{2}}\right)+t^{2} \lambda^{a b} u_{b} \frac{\partial}{\partial u_{a}}\right) .
\end{aligned}
$$

Note that symbols $X_{0}$ and $D_{0}$ has been defined in (42) and (25) and further $\psi_{\Lambda}$ is an arbitrary solution of the homogeneous equation:

$$
\left(\frac{\partial}{\partial t}-\sum_{i=1}^{m} \frac{\partial^{2}}{\partial x_{1}^{2}}\right) \psi_{\Lambda}^{j}=\Lambda^{j k} \psi_{\Lambda}^{k}
$$

\section{Equivalence transformations}

The solutions of the determining equations presented in Tables 2-5 are defined up to the equivalence transformations (26) which do not change the shape of equations (1) for arbitrary $f^{k}$. However for some particular $f^{k}$ it is possible to indicate more extended groups of equivalence transformations which include (26) as a subgroup. Here we discuss such transformations.

The most extended equivalence groups appear for the case of linear $f^{k}$ presented in Table 5. Let the related solutions have the following general form

$$
f^{k}=\Lambda^{k b} u^{b}
$$


where $\Lambda^{k b}$ is a matrix which commutes with $\left(A^{-1}\right)^{k b}$. So there exist an additional equivalence transformation

$$
u_{a} \longrightarrow \exp \left(-t \hat{\Lambda}^{c b} u_{b} \frac{\partial}{\partial u_{c}} u_{a}\right)=\exp \left(-t \hat{\Lambda}^{a b}\right) u_{b}
$$

where $\hat{\Lambda}^{c b}$ is a matrix which commutes with $A^{a b}$ and $\Lambda^{a b}$. The transformed equation has the form (45) where $f^{\prime k}=\left(\Lambda^{k b}-\hat{\Lambda}^{k b}\right) u_{b}$. In particular we can choose $\hat{\Lambda}^{k b}=\Lambda^{k b}$ and reduce the related $f^{k}$ to zero.

For $A$ diagonal there exist also the following equivalence transformation

$$
\begin{aligned}
& u_{1} \rightarrow u_{1} \exp (-k t), \quad u_{2} \rightarrow u_{2}+n t, \\
& u_{1} \rightarrow u_{1}-t u_{2}+\frac{t^{2}}{2} p, \quad u_{2} \rightarrow u_{2}-t p, \\
& u_{1} \rightarrow u_{1}+\frac{p t^{2}}{2}, \quad u_{2} \rightarrow u_{2}+p t
\end{aligned}
$$

moreover, (48) is valid only for the case when $A$ is proportional to the unit matrix.

Solutions present in Table 5 are defined up to equivalence transformations (26), (46) (49).

Using the transformation

$$
u_{1} \rightarrow \exp (n t) u_{1}, \quad u_{2} \rightarrow \exp \left(-\frac{q n}{r}\right) u_{2}
$$

it is possible to reduce to zero the parameter $n$ in Table 3, Item 1 (we will refer to this case as [T3.1]). The transformations $u_{1} \rightarrow u_{1}, u_{2} \rightarrow u_{2}+\nu t+\mu x^{2}$ enable to make zero parameters $C_{1}$ and $C_{2}$ in the cases when $f^{1}$ and $f^{2}$ have the form $f^{1}=\varphi_{1}+C_{1}, f^{2}=\varphi_{2}+C_{2}$ where $\varphi_{1}$ and $\varphi_{2}$ are given functions of $u_{1}$. Such transformations can be applied in the cases [T3.10] and [T3.8] (in the last case it is necessary to change roles of $u_{1}$ and $u_{2}$ ).

Transformations $u_{2} \rightarrow \exp (-n t) u_{2}$ and $u_{2} \rightarrow \exp (-b t) u_{2}$ give rise to new $n$ and $b$ in solutions 2 and 3 from Table 4 respectively. The transformation

$$
u_{1} \rightarrow u_{1} \exp (-s t), \quad u_{2} \rightarrow u_{2}+\frac{s r}{k} t
$$

reduces to zero parameter $s$ in non-linearities [T3.6].

Consider further the scale transformation

$$
u_{1} \rightarrow \mu u_{1}, \quad u_{2} \rightarrow \nu u_{2}, \quad t \rightarrow \lambda t \quad x \rightarrow \sqrt{\lambda x} .
$$

Under obvious conditions for the parameters defining the functions $f_{1}$ and $f_{2}$ transformations (50) enable the reduction of nonzero coefficients $p$ and $q$ to zero coefficients for solutions 1, 8, and 9 from Table 4.

We see that using equivalence transformations it is possible to make "cosmetic" improvements to the solutions found for $f^{k}$. These transformations were not used systematically because they do not change the principal classes of solutions. Also in some cases their use would complicate the presentation of the results in standard form and make them less convenient for applications. 


\section{Discussion}

In this paper we have found all possible versions of systems of diffusion equations that admit a nontrivial Lie symmetry. These results can be used to construct mathematical models with required symmetry properties in for example, physics, biology, chemistry.

In the case when the matrix $A$ is proportional to the unit matrix $I$ equation (24) admits nontrivial symmetries for all $f^{1}, f^{2}$ given in Tables $2-4$. In other words in this case we have obtained the richest spectrum of possible symmetries. If $A$ is not proportional to $I$ the number of symmetries is sufficiently reduced by the requirement of commutativity of $A^{-1}$ with the chosen matrices $B$ and $F(43)$.

In the particular case when matrix $A$ has the form $I b$ from (25) our results can be compared with those of [12]. Our results are quite similar. However, a number of our solutions, namely, seven of those presented in Table 4, Case 1 (which correspond to symmetries $Z_{N}$ ), solutions [T4.3] for $b=k=q=0$, [T5.2], [T3.11], [T3.12] and all solutions [T4.4], [T4.5], [T4.7] are missing in [12]. In addition, [T2.17] are presented in [12] incorrectly (see Table 5, item 9 here).

Consider the examples of reaction diffusion equations mentioned above in Section 1.

- The activator-inhibitor reaction equations [1] are given by

$$
\dot{u}_{1}-\frac{\partial^{2} u_{1}}{\partial x^{2}}=\frac{u_{1}^{2}}{u_{2}}-b u_{1}, \quad \dot{u}_{2}-a \frac{\partial^{2} u_{2}}{\partial x^{2}}=u_{1}^{2}-u_{2}
$$

and these are a particular case of equation (24) with the non-linearities given in $[\mathrm{T}$ $2.5]$ with $d=2, k=0, \varphi_{1}=\frac{u_{1}^{2}}{u_{2}}-b, \varphi_{2}=\frac{u_{1}^{2}}{u_{2}}-1$, and so admits the symmetry:

$$
X=X_{0}+\alpha\left(u_{1} \frac{\partial}{\partial u_{1}}+2 u_{2} \frac{\partial}{\partial u_{2}}\right)
$$

- The primitive predator-prey system can be defined by [1]

$$
\dot{u}_{1}-D \frac{\partial^{2} u_{1}}{\partial x_{2}}=-u_{1} u_{2}, \quad \dot{u}_{2}-\lambda D \frac{\partial^{2} u_{2}}{\partial x^{2}}=u_{1} u_{2}
$$

This is a particular case of (24) with non-linearities [T2.1] where $d=1, k=1, \varphi_{2}=$ $-\varphi_{1}=\frac{u_{2}}{u_{1}}$, and so it admits the symmetry:

$$
X=X_{0}+\alpha\left(D_{0}-2 u_{1} \frac{\partial}{\partial u_{1}}-2 u_{2} \frac{\partial}{\partial u_{2}}\right) .
$$

- The $\lambda-\omega$ reaction-diffusion system

$$
\dot{u}_{1}=D \Delta u_{1}+\lambda(R) u_{1}-\omega(R) u_{2}, \quad \dot{u}_{2}=D \Delta u_{2}+\omega(R) u_{1}+\lambda(R) u_{2},
$$

where $R^{2}=u_{1}^{2}+u_{2}^{2}$, and $\Delta$ is the Laplacian operator has symmetries that were analyzed in paper [2]. Again we recognize that this system is a particular case of 
(24) with non-linearities [T2.6] with $n=0$. Hence it admits the five dimensional Lie algebra generated by:

$$
X=X_{0}+\alpha\left(u_{1} \frac{\partial}{\partial u_{2}}-u_{2} \frac{\partial}{\partial u_{1}}\right)
$$

which is in accordance with results of paper [2] for the case when functions $\lambda$ and $\omega$ are arbitrary. Moreover, using Table 3, Case [T3.2] we find that for the cases when

$$
\lambda=\kappa_{1} R^{r}, \quad \omega=\kappa_{2} R^{r}
$$

equation (51) admits additional symmetry with respect to scaling transformations given by the operator:

$$
X=X_{0}+\alpha\left(u_{1} \frac{\partial}{\partial u_{2}}-u_{2} \frac{\partial}{\partial u_{1}}\right)+\nu D_{5}
$$

- The nonlinear Schrödinger equation in $m$-dimensional space is given by:

$$
\left(i \frac{\partial}{\partial t}-\Sigma_{i=1}^{m} \frac{\partial^{2}}{\partial x_{i}^{2}}\right) \psi=F\left(\psi, \psi^{*}\right) \psi
$$

also is a particular case of (24). If we denote

$$
u_{1}=\frac{1}{2}\left(\psi+\psi^{*}\right), \quad u_{2}=\frac{1}{2 i}\left(\psi-\psi^{*}\right)
$$

then (55) reduces to the form (24) with $A=-\sigma_{2}$ and

$$
f^{1}=\frac{1}{2}\left(F^{*}+F\right) u_{2}+\frac{1}{2 i}\left(F-F^{*}\right) u_{1}, \quad f^{2}=\frac{1}{2 i}\left(F-F^{*}\right) u_{2}-\frac{1}{2}\left(F+F^{*}\right) u_{1} .
$$

In other words, any solution given in Table 2 with matrices belonging to Classes $I$ $(d=0)$ and $I I$, and solutions given in Tables 3-5 with matrices belonging to Class II give rise to the non-linearity

$$
F=\frac{1}{R^{2}}\left(u_{2} f^{1}-u_{1} f^{2}+i\left(u_{2} f^{2}+f^{1} u^{1}\right)\right)
$$

for the nonlinear Schrödinger equation (55) that admits a nontrivial Lie symmetry.In the cases [T3.7] with $n=0$, [T3.2] for $s=l=q=0, r \neq \frac{4}{m}$ and $r=\frac{4}{m}$, [T4.8] for $k_{1}=n_{1}=k_{0}=0, p=q$ we recognize the well-known non-linearities [11]

$$
F=F\left(\psi^{*} \psi\right), \quad F=\left(\psi^{*} \psi\right)^{k}, \quad F=\left(\psi^{*} \psi\right)^{\frac{2}{m}}, \quad F=\ln \left(\psi^{*} \psi\right)
$$

which correspond to extended symmetries. Our analysis makes it possible to describe all other possible versions of the nonlinear Schrödinger equation with a nontrivial symmetry. We plan to discuss these elsewhere. 
Higher symmetries of the linear and nonlinear Schrödinger equations where investigated in [14], extended supersymmetries where studied in [15] The nonlinear Schrödinger equations and equations (24) for diagonal $A$ with ad hoc required symmetry with respect to the (extended) Galilei group were analyzed in [11] and [16]. We notice that the algorithm used in the present paper reduce such an analyzis to routine and simple calculations.For example, to find all systems (1) with arbitrary $n$ which are invariant with respect to the Galilei group it is sufficient to solve the system of homogeneous linear equations (31) which is easy integrated for any given invertable matrix $A$.

In the present paper we have restricted ourselves to a complete description of all possible non-linearities which generate Lie symmetry of equation (1). We have not analyzed nonclassical symmetries that may be found with using condition (13) nor have any symmetry reductions been presented. These problems will be a subject of further investigations. Finally we remark that some of the results of this paper have been presented in [17].

\section{Acknowledgement}

The authors wish to thank the Royal Society for their financial assistance for this research.

\section{References}

[1] J. D. Murray, Mathematical Biology (Springer, 1991).

[2] J. F. R. Archilla et al, J. Phys. A 39, 185 (1997).

[3] Lie S, Transformationgruppen, in 3 Bds (Liepzig, 1883).

[4] L. V. Ovsiannikov, Dokl. Acad. Nauk S.S.S.R. 123, 492 (1959).

[5] V. A. Dorodnitsyn, Comp. Meth. Phys. 22, 115 (1982).

[6] V. A. Dorodnitsyn , I .V. Kniazeva, and S. R. Svirishchevski, Diff. Uravn. 19, 1205 (1983).

[7] W. I. Fushchich and M. I. Serov, Dokl. Acad. Nauk Ukrainy 7, 23 (1990).

[8] P. A. Clarkson and E. I. Mansfield, Physica D 70, 250 (1993).

[9] R . J. Wiltshire, J. Phys. A 27, 821 (1994).

[10] W. I. Fushchych and N. I. Serov, J. Phys. A 27, L929 (1987).

[11] W. I. Fushchych, W. I. Shleten and N. I. Serov, Symmetries and Exact Solutions of Nonlinear Equations of Mathematical Physics, (Kluwer, Dordrecht, 1992).

[12] R. Cherniha and J. R. King, J. Phys. A 33, 267 (2000).

[13] P. Olver, Application of Lie groups to Differential equations (Springer-Verlag, N.Y., 1986). 
[14] W. I. Fushchych and A. G. Nikitin, J. Math. Phys. 38, 5944 (1997).

[15] J. Niederle and A. G. Nikitin, J. Math. Phys. 40, 1280 (1999).

[16] W. Fushchych and R. Cherniha, J. Phys. A 28, 5569 (1995).

[17] A. G. Nikitin and R. J. Wiltshire, in: Symmetries in Nonlinear Mathematical Physics, Proceedings of Third International Conference, Kiev, July 12-18, 1999, Institute of Mathematics of Nat. Acad. Sci. of the Ukraine (2000), pp. 47-59. 